\title{
Homeodomain Interacting Protein Kinase 2 Regulates Postnatal Development of Enteric Dopaminergic Neurons and Glia via BMP Signaling
}

\author{
Alcmène Chalazonitis, ${ }^{1 *}$ Amy A. Tang, ${ }^{2,3 *}$ Yulei Shang, ${ }^{2,3}$ Tuan D. Pham, ${ }^{1}$ Ivy Hsieh, ${ }^{2,3}$ Wanda Setlik, ${ }^{1}$ \\ Michael D. Gershon, ${ }^{1}$ and Eric J. Huang ${ }^{2,3}$ \\ ${ }^{1}$ Department of Pathology and Cell Biology, Columbia University, New York, New York 10032, ${ }^{2}$ Department of Pathology, University of California San \\ Francisco, San Francisco, California 94143, and ${ }^{3}$ Pathology Service 113B, VA Medical Center, San Francisco, California 94121
}

\begin{abstract}
Trophic factor signaling is important for the migration, differentiation, and survival of enteric neurons during development. The mechanisms that regulate the maturation of enteric neurons in postnatal life, however, are poorly understood. Here, we show that transcriptional cofactor HIPK2 (homeodomain interacting protein kinase 2) is required for the maturation of enteric neurons and for regulating gliogenesis during postnatal development. Mice lacking HIPK2 display a spectrum of gastrointestinal (GI) phenotypes, including distention of colon and slowed GI transit time. Although loss of HIPK2 does not affect the enteric neurons in prenatal development, a progressive loss of enteric neurons occurs during postnatal life in Hipk $2^{-1-}$ mutant mice that preferentially affects the dopaminergic population of neurons in the caudal region of the intestine. The mechanism by which HIPK2 regulates postnatal enteric neuron development appears to involve the response of enteric neurons to bone morphogenetic proteins (BMPs). Specifically, compared to wild type mice, a larger proportion of enteric neurons in Hipk $2^{-l-}$ mutants have an abnormally high level of phosphorylated Smad1/5/8. Consistent with the ability of BMP signaling to promote gliogenesis, Hipk2 ${ }^{-1-}$ mutants show a significant increase in glia in the enteric nervous system. In addition, numbers of autophagosomes are increased in enteric neurons in $\mathrm{Hipk}^{-l-}$ mutants, and synaptic maturation is arrested. These results reveal a new role for HIPK2 as an important transcriptional cofactor that regulates the BMP signaling pathway in the maintenance of enteric neurons and glia, and further suggest that HIPK2 and its associated signaling mechanisms may be therapeutically altered to promote postnatal neuronal maturation.
\end{abstract}

\section{Introduction}

Enteric neurons are derived from precursors that originate in the neural crest, migrate to the gut, and undergo a long process of development and maturation (Burns and Pachnis, 2009; Gershon, 2010). Although a great deal is known about the early events in enteric neuron development, much less is known about later-acting mechanisms for the acquisition of enteric neuronal phenotype diversity, maturation, and survival (Enomoto et al., 1998; Flynn et al., 2007; Uesaka et al., 2007, 2008; Burns and Pachnis, 2009; Uesaka and Enomoto, 2010). Several studies indicate that bone morphogenetic proteins (BMPs), members of the

Received March 1, 2011; revised July 27, 2011; accepted July 30, 2011.

Author contributions: A.C., M.D.G., and E.J.H. designed research;A.C., A.A.T.,Y.S., T.D.P.,I.H., and W.S. performed research; Y.S. contributed unpublished reagents/analytic tools; A.C., A.A.T., Y.S., T.D.P., I.H., W.S., M.D.G., and E.J.H. analyzed data; A.C., M.D.G., and E.J.H. wrote the paper.

This work was supported by grants from National Institutes of Health Grants NS48393 and RR24858 (to E.J.H.) and NS12969 and NS15547 to (M.D.G.) and by Department of Veterans Affairs Merit Review Award (to E.J.H.). We thank Dr. Moses Chao for the TrkC antibody, members of the Huang Laboratory and Gershon Laboratory for many helpful discussions, and Dr. David Sulzer, Department of Neurology, Columbia University, for helpful comments on the manuscript.

${ }^{*}$ A.C. and A.A.T. contributed equally to this work.

Correspondence should be addressed to Eric J. Huang, Department of Pathology, University of California San Francisco, Health Sciences West, Room 450E, 513 Parnassus Avenue, San Francisco, CA 94143-0506. E-mail: eric.huang2@ucsf.edu.

DOI:10.1523/JNEUROSCI.1078-11.2011

Copyright $\odot 2011$ the authors $\quad 0270-6474 / 11 / 3113746-12 \$ 15.00 / 0$ transforming growth factor $\beta$ (TGF $\beta$ ) superfamily, regulate the development of enteric neurons (Chalazonitis et al., 2004, 2008; Fu et al., 2006). Interestingly, the effects of BMPs on enteric neurons are context dependent; outcome is dictated by the timing of BMP signals and the interaction between BMPs and their antagonist, Noggin. BMPs, for example, promote the development of the neurotrophin-3 (NT-3)-dependent, TrkC+ enteric dopaminergic neurons. In contrast, transgenic mice expressing Noggin under the control of the neuron-specific enolase (NSE) promoter have reduced numbers of enteric dopaminergic neurons (Chalazonitis et al., 2008). These results support the idea that BMP signaling is essential for subtype specification in the enteric nervous system (ENS) and provide important insights into the control of maturation, survival, and long-term maintenance of enteric dopaminergic neurons ( $\mathrm{Li}$ et al., 2004, 2006). Moreover, increased BMP signaling promotes glial differentiation in cultured enteric crest-derived precursors and in NSE-BMP4 transgenic mice (Chalazonitis et al., 2011).

The signal transduction pathways of TGF $\beta$ and BMP involve the Smad proteins, which are highly evolutionarily conserved transcription factors that are activated upon the engagement of TGF $\beta$ or BMPs with their receptors (Derynck and Zhang, 2003; Massague et al., 2005). Following their phosphorylation, Smads are translocated to the nucleus where they regulate the expression of TGF $\beta(\operatorname{Smad} 2 / 3)$ or BMP $(\operatorname{Smad} 1 / 5 / 8)$ target genes. In addi- 
tion, Smads can interact with a number of transcriptional coactivators or corepressors to activate or silence the transcription of target genes, thus adding layers of complexity to the final outcomes of TGF $\beta$ or BMP signals. HIPK2 (homeodomain interacting protein kinase 2) can negatively regulate BMP-dependent reporter gene activity in cell lines and interact with Smad1, Smad2, Smad3, and c-Ski (Harada et al., 2003). HIPK2 can also regulate survival and apoptosis in sensory, sympathetic, and midbrain dopamine (DA) neurons (Doxakis et al., 2004; Wiggins et al., 2004; Zhang et al., 2007). The present study focuses on the role of HIPK2 in the maturation of enteric neurons and, in particular, the dopaminergic subset. These neurons were chosen because they play an important role in regulating intestinal peristalsis (Li et al., 2004, 2006) and may be affected in Parkinson's disease (Kuo et al., 2010). Our data indicate that enteric neurons require HIPK2 in postnatal life for the formation of autophagosomes and for synaptogenesis. These results underscore the important function of HIPK2 in regulating BMP signaling in the maturation of enteric neurons, especially those of the dopaminergic subtype.

\section{Materials and Methods}

Animals. The Hipk2 ${ }^{+/-}$mutants were maintained on a mixed 129; C57BL/6 background. The procedures used to generate and genotype Hipk $2^{-1-}$ mutants have been described previously (Wiggins et al., 2004; Zhang et al., 2007). Animal care was approved by the Institutional Animal Care and Use Committee at University of California at San Francisco and Columbia University and followed National Institutes of Health guidelines.

Cuprolinic blue labeling and acetylcholinesterase histochemistry. Both wild type and Hipk $2^{-1-}$ mutant mice of either sex ranging in age from 10 to 24 weeks were perfused with $4 \%$ paraformaldehyde (PFA). The submucosal and myenteric plexuses were dissected separately. Laminar preparations of the gut wall were prepared as whole mounts to include either the submucosa with the submucosal plexus or the myenteric plexus attached to the longitudinal muscle. Neurons were identified with the neuronal ribosomal marker cuprolinic blue as described previously (Heinicke et al., 1987; Karaosmanoglu et al., 1996; Chalazonitis et al., 2004; Chalazonitis et al., 2008). Regions of the gut examined included stomach, duodenum, jejunum, proximal and distal ileum, and colon.

Acetylcholinesterase (AChE) activity was demonstrated histochemically according to procedures described previously (Enomoto et al., 1998). Briefly, the gut was dissected as a single piece from the proximal esophagus to the distal colon; mesenteric attachments and pancreas were removed, and the tissue was postfixed with $4 \%$ PFA for $1-2 \mathrm{~h}$ at $4^{\circ} \mathrm{C}$. Tissues were then transferred to saturated sodium sulfate and stored overnight at $4^{\circ} \mathrm{C}$. Preparations were then incubated in buffer $(0.2 \mathrm{~mm}$ ethopropazine $\mathrm{HCl}, 4 \mathrm{~mm}$ acetylthiocholine iodide, $10 \mathrm{~mm}$ glycine, $2 \mathrm{~mm}$ cupric sulfate, and $65 \mathrm{~mm}$ sodium acetate, $\mathrm{pH} 5.5$, for 2-4 h. Staining for acetylcholinesterase was developed by incubation in sodium sulfide for $1.5 \mathrm{~min}(1.25 \%, \mathrm{pH} 6)$. Tissue was rinsed extensively with water before photography under a dissecting microscope. Photomicrographs were captured using an Olympus BX51 microscope connected to a DP70 CCD camera and DP Manager 3.1.1 software. For pictures, the gut was opened along the mesenteric border, flattening the tissue with the serosal side up, and mounted in 50\% glycerol. Images obtained in the Gershon Laboratory were captured with a Leica DML6000B microscope connected to a Retiga Exi CCD camera using Improvision Volocity 5.4.1 software.

Immunohistochemistry. HIPK2 protein and HIPK2 ${ }^{\text {LacZ }}$ were detected using procedures described previously (Wiggins et al., 2004; Zhang et al., 2007). To identify DA neurons in the ENS, segments of duodenum, jejunum, ileum, and colon were dissected to expose the myenteric or submucosal plexuses in laminar preparations. Before labeling with antibodies to dopamine, the tissue was exposed for $24 \mathrm{~h}$ to a fixative modified to preserve tissue DA that included $4 \%$ PFA in $0.1 \mathrm{~m}$ phosphate buffer saline, $9 \%$ sucrose, and $0.025 \% \mathrm{~L}$-ascorbic acid, $\mathrm{pH}$ 7.0. Following a $5 \mathrm{~h}$ rinse in $0.1 \mathrm{~m}$ phosphate buffer, the tissue was incubated with rat anti- bodies to DA (1:1000, NT-104, Protos Biotech) for an additional $24 \mathrm{~h}$ at $4^{\circ} \mathrm{C}$. Following rinses in PBS, laminar preparations were incubated for $4 \mathrm{~h}$ with donkey secondary antibodies to rat IgG conjugated to Alexa Fluor 594 (1:750, Invitrogen), rinsed, and mounted with Vectashield (Vector Laboratories). Because DA neurons are relatively rare in the myenteric plexus but more abundant in the submucosal plexus (Li et al., 2004; Chalazonitis et al., 2008), quantitative analyses were limited to the laminar preparations of submucosal plexus. Double immunolabeling of DA and tyrosine hydroxylase (TH) was carried out to verify the coincident location of these two antigens in the same neurons.

The primary antibodies in this study included the following: a polyclonal sheep anti-tyrosine hydroxylase antibody (1:200, P60101, PelFreeze); anti-BrdU antibody (1:200, MAB3228, Millipore); anti-NF150 antibody (1:400, AB1981, Millipore); anti-cleaved caspase 3 antibody (1:1000, AF835, R \& D Systems); anti-HuC/D antibody (1:20, A-21271, Invitrogen); anti-LC3 antibody (1:50, M152-3, Medical and Biological Laboratories); anti-PGP9.5 antibody (1:20, ab8189, Abcam); antiSNAP25 antibody (1:20, ab41455, Abcam); anti-HIPK2 antibody (1:500, catalog no. ARP32586-P050, Aviva); anti-GFR $\alpha 1$ (1:1000, AB5963, Millipore Bioscience Research Reagents); anti-PSD95 antibody (1:100, catalog no. 2507, Cell Signaling Technology); anti-p75 ${ }^{\text {NTR }}$ antibody (1: 2500, G3231, Promega); anti-synapsin antibody (1:20, catalog no. 2312, Cell Signaling Technology); TuJ1 anti-class III $\beta$-tubulin antibody (1: 1000, MMS-435P, Covance); anti-pSmad1/5/8 antibody (1:625, catalog no. 9511, Cell Signaling Technology); anti-pSmad2 antibody (1:200, catalog no. 3010, Cell Signaling Technology); anti-TrkC antibody (gift from Dr. Moses Chao, Skirball Institute, New York University); and antiS100 $\beta$ antibody (1:3000, catalog no. Z0311, DakoCytomation). The specificity of the anti-HIPK2 antibody was confirmed by the lack of staining dorsal root ganglion neurons in Hipk2 ${ }^{-1-}$ mutant mice (data not shown). Secondary antibodies used included donkey anti sheep IgG coupled to Alexa Fluor 488 (1:500; Invitrogen), anti-rabbit IgG-conjugated with HRP (Vector Laboratories), and goat anti rabbit antibody coupled to Alexa Fluor 594 (1:625; Invitrogen).

TUNEL in enteric neurons. Following detection of enteric neurons with TuJ1 antibodies, tissue sections were incubated in labeling buffer for $3 \mathrm{~min}$ at room temperature, followed by the addition of terminal deoxynucleotidyl transferase (TdT) reaction mix ( $4 \mu$ l of deoxyribonucleotide triphosphate mix, $4 \mu \mathrm{l}$ of $50 \times \mathrm{Mn}^{2+}$ stock solution, $4 \mu \mathrm{l}$ of TdT enzyme, and $50 \mu \mathrm{l}$ of TdT labeling buffer) (R \& D Systems) in a humidified chamber at $37^{\circ} \mathrm{C}$ for $1.5 \mathrm{~h}$. The reaction was terminated by incubating the slides with $1 \times$ stop buffer for $5 \mathrm{~min}$, and the slides were rinsed twice in PBS for $2 \mathrm{~min}$. To visualize the biotinylated nucleotides, which have been transferred by the TdT enzyme onto the nicked ends of single-strand DNA, samples were incubated in Strep-Fluor solution at room temperature for $1 \mathrm{~h}$. Each slide was then drained and mounted with 50-75 $\mu \mathrm{l}$ of anti-Fade mounting media.

Electron microscopy. Mice were fixed by perfusion at postnatal day $(\mathrm{P})$ $0, \mathrm{P} 7, \mathrm{P} 16$, and P36 with a freshly prepared mixture of $4 \%$ formaldehyde (from paraformaldehyde) and $0.2 \%$ glutaraldehyde in $0.1 \mathrm{M}$ phosphate buffer, $\mathrm{pH}$ 7.4. Segments of stomach, duodenum, ileum, and colon were dissected and postfixed in $4 \%$ formaldehyde (from paraformaldehyde) and $2.5 \%$ glutaraldehyde in the same buffer overnight at $4^{\circ} \mathrm{C}$. Gut segments were treated with $2 \%$ osmium tetroxide for $1 \mathrm{~h}$ at room temperature, rinsed 5 times in PBS and maleate buffer, and stained en bloc with $5 \%$ aqueous uranyl acetate on ice for $1 \mathrm{~h}$. The blocs of tissue were dehydrated with a graded series of ethanol solutions, cleared with propylene oxide, and embedded in polymerized EMbed-812 (Ted Pella). Ultra thin sections (1 micron) were cut with a microtome (Reichert-Jung, Reichert) and collected on copper grids. Sections were examined with a JEOL 1200EX electron microscope.

Measurements of gut motility. Gut motility was measured using a protocol modified from Carai et al. (2006). Six wild type and five Hipk2 ${ }^{-1-}$ mice of either sex, 8-12 weeks of age, were used for this study. Mice were housed individually during the course of the study and were not deprived of food before measurements of total gastrointestinal transit time. Xylene cyanol FF and bromophenol blue $(0.25 \% \mathrm{w} / \mathrm{v}$, each) were suspended in water containing $0.5 \%$ methylcellulose and administered intragastrically with a feeding needle ( 25 gauge, 1 inch long, $1.25 \mathrm{~mm}$ ball diameter) at a 
dose of $0.3 \mathrm{ml}$ per mouse. Beginning immediately after Xylene cyanol FF and bromophenol blue administration, fecal pellets were collected in preweighed tubes to determine wet weights every $30 \mathrm{~min}$ for up to $6 \mathrm{~h}$. To determine total gastrointestinal transit time (GITT), mice were monitored $8 \mathrm{~h}$ after Xylene cyanol FF and bromophenol blue administration at $30 \mathrm{~min}$ intervals. The time required for the blue bolus to disappear and the first brown bolus to appear was recorded as GITT. Fecal pellets were vacuum dried overnight to determine dry weights. Water content in fecal pellets was determined as the difference between the wet and dry weight of stool.

Quantification and statistical analyses. Neuronal and glial cell bodies were counted using a $40 \times$ objective in 10 contiguous nonoverlapping rectangular fields covering $1.254 \mathrm{~mm}^{2}$ of plexus and counted as one measurement. The investigator counting neurons was blinded with regard to the genotype of the animals. The numbers of measurements obtained from each region of the gut and in each plexus using cuprolinic blue as a neuronal marker are listed in Table 1. The incidence of autophagy was estimated as a percentage of identified autophagosome profiles normalized to $10^{-3} \mu \mathrm{m}^{2}$ unit area of plexus. Sections from Hipk $2^{-1-}$ and wild type mice were examined similarly and compared. The number of synapses in the myenteric plexus was determined by enumerating structures that contain well delineated presynaptic buttons, synaptic vesicles, synaptic clefts, and postsynaptic densities. The synaptic density was calculated based on the number of synapses normalized to $10^{-3} \mathrm{~mm}^{2}$ unit area. Means \pm SEM were calculated and significance was analyzed by Student's $t$ test (between 2 means of values) or analysis of variance (ANOVA; Tukey or Bonferroni-Dunn post hoc comparison) with $>2$ means of values.

\section{Results}

\section{Perinatal lethality and bowel motility} abnormalities in Hipk $2^{-/-}$mutants Homozygous Hipk2 (Hipk2 ${ }^{-1-}$ ) mutants were born in a normal Mendelian ratio and displayed no gross abnormalities; nevertheless, $\sim 40 \%$ of Hipk $2^{-/-}$mutants died before weaning, most within 2-3 d after birth (Fig. 1A). In contrast, heterozygous Hipk2 (Hipk2 $\left.{ }^{+/-}\right)$mice survived into adulthood just as their wild type littermates did. Of those Hipk2 $2^{-/-}$mutants that died within $3 \mathrm{~d}$ after birth, almost all lacked milk in the stomach (Fig. 1B, top). At necropsy, the small intestines were distended and contained large amounts of air (Fig. $1 \mathrm{~B}$, bottom). The remaining $60 \%$ of Hipk $2^{-1-}$ mutants survived into adulthood but gained weight poorly and remained abnormally small (Fig. 1C). By 12 weeks of age, the body weight of Hipk2 $2^{-1-}$ mutants was only $70 \%$ of that of wild type (24.2 $\mathrm{g}$ in wild type vs $17.2 \mathrm{~g}$ in Hipk $2^{-1-}$ mutants) (Fig. $1 C$ ). In contrast, the body length of Hipk2 ${ }^{-1-}$ mutants, measured by the distance from nose to anus, did not differ significantly from that of wild type mice (data not shown). Importantly, there was no evidence that the gastrointestinal phenotypes were caused by congenital malformations, such as tracheoesophageal fistula.

The distended bowel in Hipk $2^{-1-}$ mutants at perinatal stages and poor body weight gain in postnatal life suggested that
Table 1. The number of measurement for the neuronal cell body in the myenteric and submucosal plexuses in wild type and Hipk2 ${ }^{-1-}$ mutant mice

\begin{tabular}{|c|c|c|c|c|}
\hline \multirow[b]{2}{*}{ Gut regions } & \multicolumn{2}{|l|}{ Wild type } & \multicolumn{2}{|c|}{ Hipk2 ${ }^{-I}$ mutants } \\
\hline & $\operatorname{Mice}^{\dagger}(N)$ & Measurements $(n)^{*}$ & $\operatorname{Mice}^{\dagger}(M)$ & Measurements $(n)^{*}$ \\
\hline
\end{tabular}

Myenteric plexus

$\begin{array}{lllll}\text { Stomach } & 2 & 18 & 2 & 18 \\ \text { Duodenum } & 8 & 59 & 8 & 59 \\ \text { Jejunum } & 7 & 57 & 7 & 45 \\ \text { Proximal ileum } & 5 & 29 & 5 & 30 \\ \text { Distal ileum } & 3 & 18 & 3 & 18 \\ \text { Distal colon } & 4 & 24 & 4 & 24 \\ \text { Submucosal plexus } & & & & \\ \text { Duodenum } & 8 & 59 & 8 & 54 \\ \text { Jejunum } & 7 & 52 & 7 & 45 \\ \text { Proximal ileum } & 5 & 28 & 5 & 24 \\ \text { Distal ileum } & 3 & 14 & 3 & 18 \\ \text { Distal colon } & 4 & 21 & 4 & 24\end{array}$

*Each measurement corresponded to values summed from scanning 10 microscope fields with a $40 \times$ objective. ${ }^{\dagger}$ The specimens were collected from wild type and Hipk $2^{-1-}$ mutant mice of either sex at 10, 12, 16, 21, and 24 weeks of age.
A

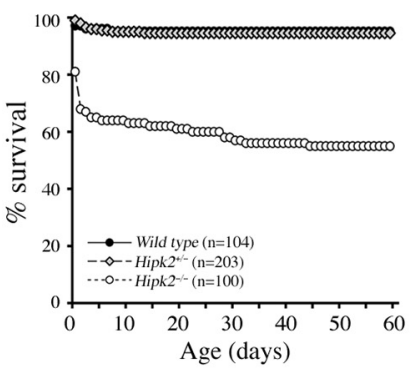

C

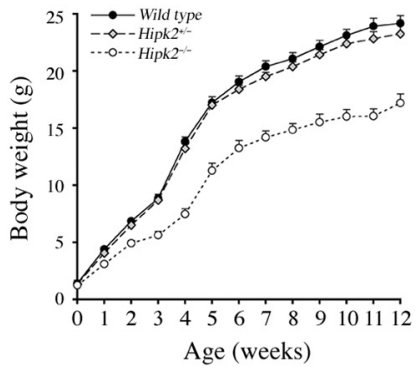

E

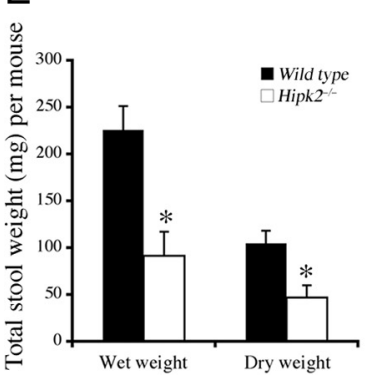

B

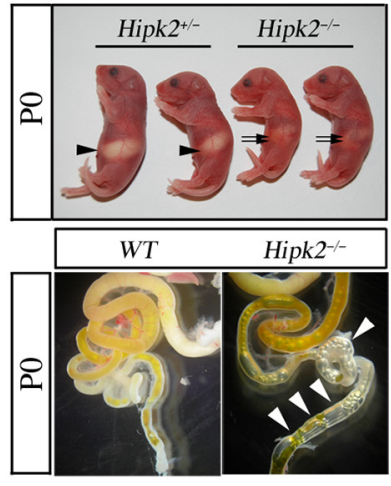

D

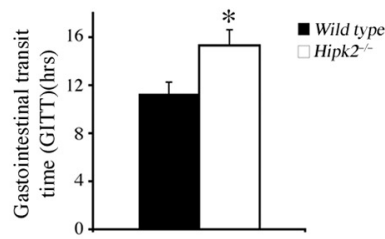

Figure 1. Perinatal lethality and gastrointestinal phenotype in Hipk $2^{-1-}$ mutant mice. $\boldsymbol{A}-\boldsymbol{B}$. More than $40 \%$ of Hipk $2^{-1-}$ mutant mice in the mixed 129;B6 background die in postnatal life, with the majority within the first $2 \mathrm{~d}$ of life (A). Most of the dying Hipk2 ${ }^{-1-}$ mutants show no evidence of milk in the stomach (highlighted by arrows) (B, top). In contrast, the wild type littermates show milk in the stomach ( $\boldsymbol{B}$, top, arrowheads). Necropsy of Hipk $2^{-1-}$ mutant mice at P0 show slightly distended intestinal tract filled with gas ( $\boldsymbol{B}$, bottom, arrowheads). $\boldsymbol{C}$, Compared to the normal weight gains for wild type mice in the first 12 weeks in postnatal life, Hipk $2^{-1-}$ mutant mice of either sex show reduce body weight gain without affecting the body length (data not shown). D-G, Adult Hipk $2^{-1-}$ mutant mice $(8-12$ weeks old) show prolonged gastrointestinal transit time $(\boldsymbol{D})$, reduced stool output $(\boldsymbol{E}, \boldsymbol{F})$, and reduced water content in the stool $(\boldsymbol{G})$. Student's $t$ test, $n=6$ for wild type and Hipk $2^{-I-}$ mutants; ${ }^{*} p<0.05$. 


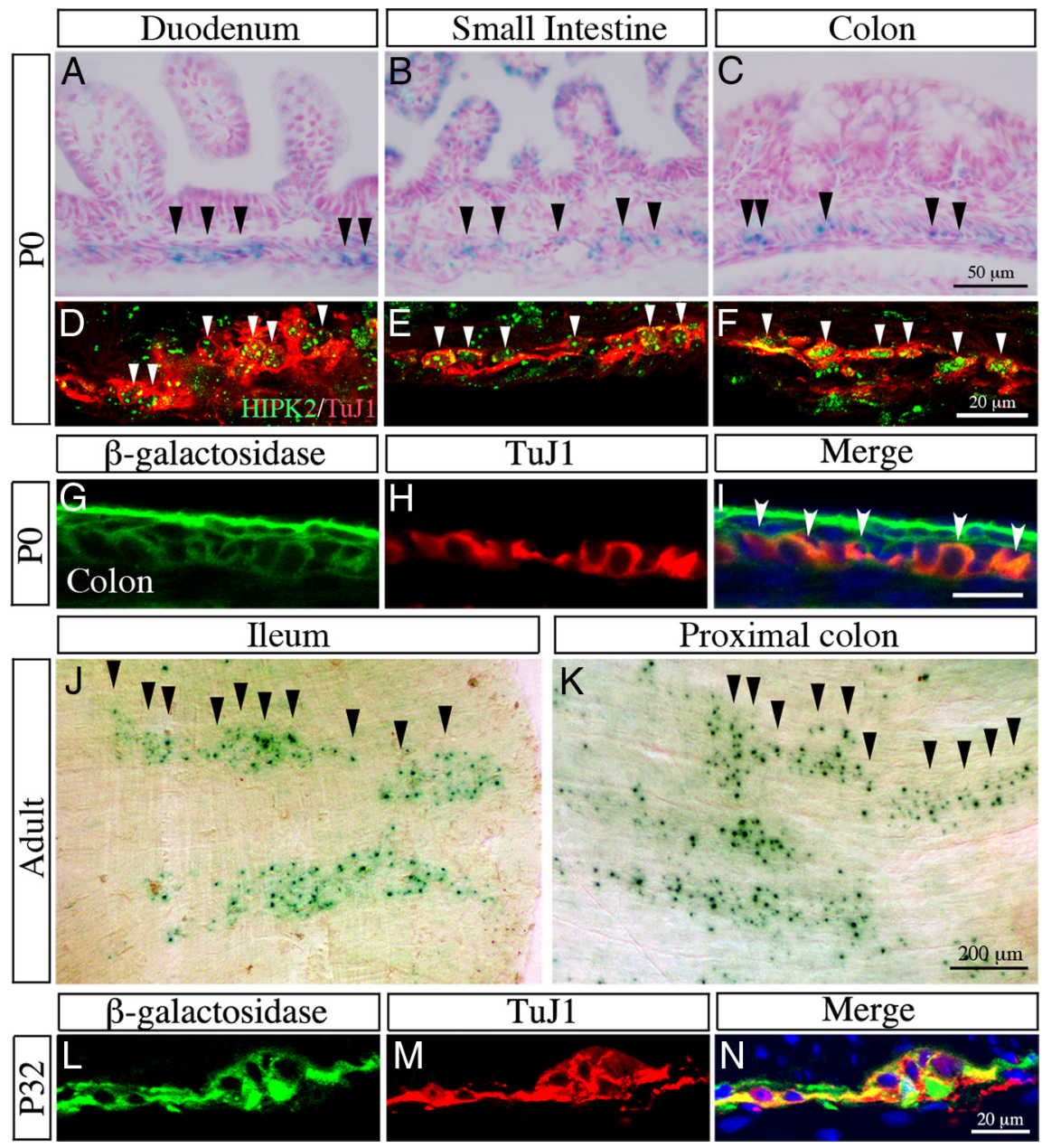

Figure 2. Expression of HIPK2 in the enteric neurons in postnatal stages. $\boldsymbol{A}-\boldsymbol{C}$, The expression of HIPK2 can be detected using HIPK2 ${ }^{\text {LacZ }}$ in the myenteric plexus in duodenum (A), small intestines (B), and colon (C). Scale bar: (in C) $\boldsymbol{A}-\mathbf{C}, 50 \mu \mathrm{m}$. D-I, Confocal images indicate that the endogenous HIPK2 protein $(\boldsymbol{D}-\boldsymbol{F})$, detected using anti-HIPK2 antibody, and HIPK2 ${ }^{\text {LacZ }}(\mathbf{G}-\boldsymbol{I})$, detected using anti- $\beta$-galactosidase antibody, show extensive colocalization with neuronal marker TuJ1. Note that HIPK2 protein ( $\boldsymbol{D}-\boldsymbol{F}$, in green channel) is present as puncta in the nuclei of enteric neurons (TuJ1+, red channel). Background staining in the green channel is detected in the fibrous tissue at the serosal layer $(\mathbf{G}, \boldsymbol{I})$. Scale bar: (in $\boldsymbol{I}) \mathbf{D}-\boldsymbol{I}, 20 \mu \mathrm{m}$. J-N, Expression of HIPK2 in the enteric neurons of adult mice can also be detected using whole-mount staining for HIPK2 ${ }^{\text {Lac2 }}$ in the myenteric plexus in ileum and proximal colon. Note the distinct aggregates of HIPK2 ${ }^{\text {LacZ }}$ in the myenteric plexus (highlighted by arrowheads) surrounded by light green diffusion of HIPK2 ${ }^{\text {LacZ }}$ activity into adjacent tissue $(\boldsymbol{J}, \boldsymbol{K})$. Confocal microscopy shows extensive colocalization of HIPK2 ${ }^{\text {LacZ }}$ with TuJ1 at P32 ( $\boldsymbol{L}-\boldsymbol{N}$ ). Scale bars: (in $\boldsymbol{K}) \boldsymbol{J}, \boldsymbol{K}, 200 \mu \mathrm{m}$; (in $\boldsymbol{N}) \boldsymbol{L}-\boldsymbol{N}, 20 \mu \mathrm{m}$.

GI motility might be abnormal. To test this hypothesis, the gastrointestinal transit time was determined in wild type and Hipk2 ${ }^{-1-}$ mutants. Results indicated that GITT was significantly longer in Hipk $2^{-l-}$ mutants than in wild type mice $(15.3 \pm 1.3 \mathrm{~h}$ vs $11.3 \pm$ $0.9 \mathrm{~h}, n=6, p<0.05$ ) (Fig. $1 D$ ). In addition, under normal feeding conditions the $24 \mathrm{~h}$ stool quantity and the stool pellet size of Hipk $2^{-1-}$ mutants were much smaller than those of wild type mice, regardless of stool water (Fig. $1 E, F$ ). The water content of the stools of Hipk $2^{-1-}$ mutants, furthermore, was only $50 \%$ of that of wild type mice (Fig. 1G).Together, these results suggest that the gastrointestinal motility is abnormal in Hipk $2^{-1-}$ mutants, which accounts for the distended bowel of neonates and the constipation of adult mice.

Loss of neurons in myenteric and submucosal plexuses in Hipk $2^{-/-}$mutants

To investigate the mechanism of the abnormal gastrointestinal (GI) transit of Hipk2 ${ }^{-1-}$ mutants, we characterized the expres- sion of HIPK2 in the ENS. Using $H$ ipk $2^{\text {LacZ }}$ as a reporter, we detected scattered HIPK2 expression in neurons along the wall of small and large intestines as early as embryonic day (E) 15.5 (data not shown). The HIPK2 ${ }^{\text {LacZ }}$ expression persisted in the enteric neurons through $\mathrm{P} 0$ (Fig. 2A-C,G-I). Consistent with these results, the endogenous HIPK2 protein was detected using a HIPK2-specific antibody in speckles within the nuclei of enteric neurons (Fig. $2 D-F$ ). In adult mice, HIPK $2^{\text {LacZ }}$ showed extensive colocalization with clusters of neurons within the myenteric plexus in both small and large intestines (Fig. $2 \mathrm{~J}-\mathrm{N}$ ).

Several approaches were used to determine whether the loss of HIPK2 affected the number of enteric neurons. First, we characterized the development of enteric neurons during prenatal life. We used p75 ${ }^{\text {NTR }}$ as a marker for crest-derived cells, BrdU, and various neuronal markers, including $\beta 3$-tubulin, neurofilament (NF150), PGP9.5, and HuC/D, to quantify precursors and neurons in the developing gut. The numbers of crest-derived cells and neurons in the developing ENS of wild type mice were not significantly different from those of Hipk2 ${ }^{-1-}$ mutants at E15.5 or E18.5 (data not shown). Second, we used AChE activity in whole mounts to visualize the ENS in the postnatal intestine. Since $>80 \%$ of enteric neurons are cholinergic (Sang and Young, 1998; Furness, 2006) and the overwhelming majority of enteric neurons and neurites express AChE, the AChE wholemount staining provided a convenient tool to evaluate the overall organization of neuronal cell body and nerve fiber within the ENS (Blaugrund et al., 1996; Wang et al., 2010). In wild type intestine at P0, ganglia were well developed (Fig. 3A-C, arrowheads), and neural connectives were intensely stained with AChE reaction product (Fig. $3 A-C$, arrows). In contrast, the size of ganglia of the Hipk2 ${ }^{-1-}$ mutant ENS was smaller than that in wild type mice (Fig. 3D-F, arrowheads), and the density of neural connectives as well as the intensity of AChE staining was reduced (Fig. 3D-F, arrows). A similar pattern of AChE activity was also detected in adult intestines; again, the size of enteric ganglia and the density of neural connectives were noticeably reduced in Hipk $2^{-1-}$ mutants (Fig. 3J-L), compared with wild type controls (Fig. 3G-I).

The diminished AChE activity in the gut of Hipk $2^{-1-}$ mutants suggested that the number of enteric neurons might be reduced. To test this, we analyzed the packing density of enteric neurons using cuprolinic blue as a neuronal marker. The numbers of neurons in both the myenteric and submucosal plexuses in Hipk2 mutants at 12 weeks of age were significantly lower than those in wild type mice (Fig. 4A-D). Interestingly, quantification of neuronal packing density (number of neurons per unit area) in the myenteric plexus of small and large intestines of wild type and 
Hipk2 ${ }^{-1-}$ mutants showed no difference at P1. There was, however, a progressive reduction of neurons in the myenteric plexus of the Hipk2 ${ }^{-1-}$ mutant at P14 and P85 (Fig. 4E, F). By P85, the myenteric plexus of Hipk $2^{-1-}$ mutants showed a $20-33 \%$ reduction of neuronal density compared to that in wild type mice, depending upon the region of bowel analyzed (Fig. $4 F$ ). In addition, the packing density of the submucosal neurons of P85 Hipk $2^{-1-}$ mutants also was similarly lower than that of wild type mice (Fig. 4G).

\section{Loss of HIPK2 results in severe decreases of dopaminergic neurons in the ENS}

The ENS contains dopaminergic neurons that critically regulate intestinal motility (Li et al., 2004, 2006); moreover, the development of the dopaminergic neurons of the ENS is regulated by members of the TGF $\beta$ superfamily, such as BMP-2, BMP-4, and their antagonists like Noggin (Chalazonitis et al., 2008). Since our previous results had demonstrated that Hipk $2^{-1-}$ mutants lost a significant number of dopaminergic neurons in the ventral midbrain (Zhang et al., 2007), we postulated that the loss of HIPK2 might also negatively affect the development of dopaminergic neurons in the ENS. To test this hypothesis, we quantified dopaminergic neurons in the ENS using antibodies to dopamine and $\mathrm{TH}$ as markers. Duodenum, jejunum, ileum, and colon were dissected from wild type and Hipk $2^{-1-}$ littermates of either sex that were $12-24$ weeks of age ( $n=5$ for wild type and $n=6$ for Hipk ${ }^{-1-}$ mutants). The packing density of dopaminergic neurons, determined from observations of dopamine immunoreactivity alone or coincident dopamine and $\mathrm{TH}$ immunoreactivities, was quantified per $\mathrm{mm}^{2}$ of submucosal plexus. Consistent with previous studies ( $\mathrm{Li}$ et al., 2004), we found that dopaminergic neurons were most prevalent in the submucosal plexus of wild type mice, where dopamine and TH immunoreactivities were detected in the cytoplasm of the same neurons (Fig. $4 H-J$ ). In Hipk $2^{-l-}$ mutants, however, the absolute number of DA neurons was consistently reduced in the submucosal plexus of duodenum, jejunum, ileum, and colon (Fig. $4 K-M$ ). To determine whether the decrease in packing density of dopaminergic neurons reflected the decrease in the overall neuronal packing density observed in the four regions of the gut examined, we normalized the numbers of dopaminergic neurons to the numbers of total neurons in wild type and Hipk $2^{-1-}$ mutants. Whereas the normalized percentage of dopaminergic neurons was not significantly reduced in the duodenum in Hipk $2^{-1-}$ mutants, it was decreased to $72 \%$ of the wild type level in jejunum, to $53 \%$ of the wild type level in ileum, and to $45 \%$ of the wild type level in colon (Fig. 4O,P). These data suggest that, except for the duodenum, decreases in enteric dopaminergic neurons were much more severe in Hipk $2^{-1-}$ mutants relative to the total neuron numbers and thus did not simply reflect the decrease in the overall number of neurons observed in all regions of the
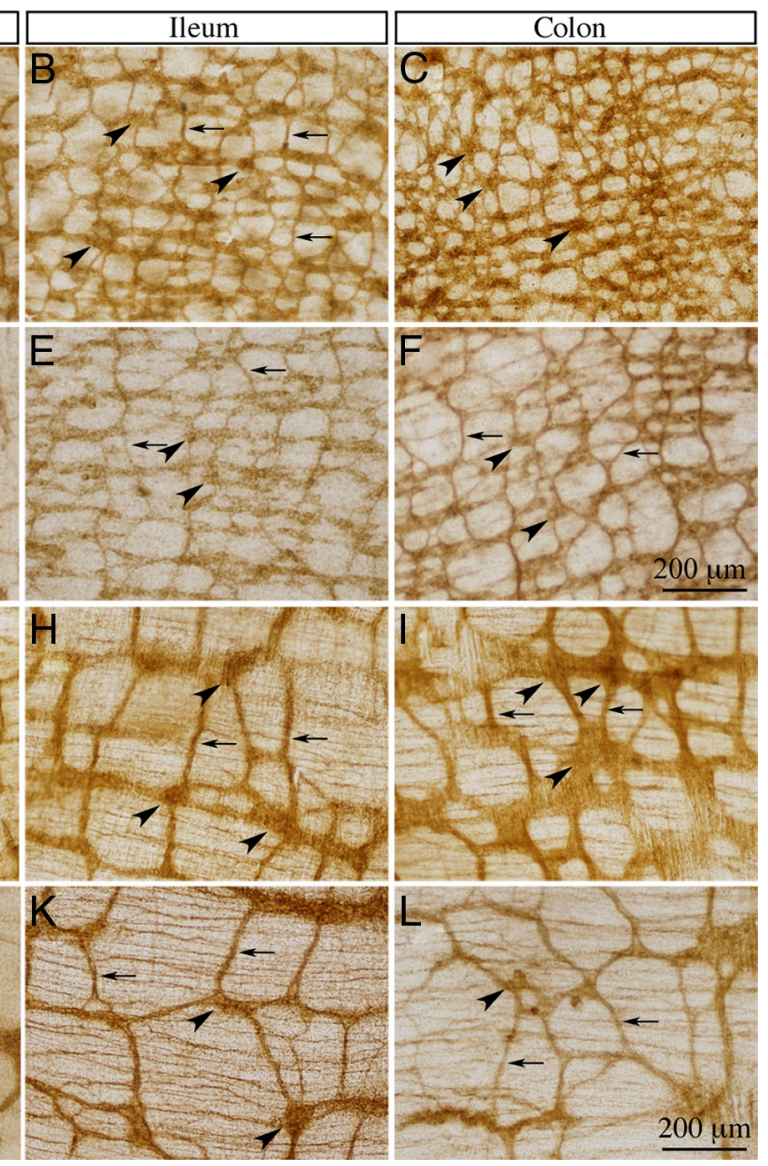

Figure 3. Reduced ganglion size and interganglionic nerve fibers in the myenteric plexus of the duodenum, ileum, and colon in Hipk $2^{-1-}$ mutants at postnatal stages. $\boldsymbol{A}-\boldsymbol{F}$, Whole-mount AChE staining of the duodenum, ileum, and proximal colon show reduced size in the myenteric plexus (arrowheads) and reduced nerve fiber staining intensity in Hipk2 ${ }^{-1-}$ mutants (arrows) as early as P0. Scale bar: (in $\boldsymbol{F}) \boldsymbol{A}-\boldsymbol{F}, 200 \mu \mathrm{m}$. $\mathbf{G}-\boldsymbol{L}$. The reduction in the size of myenteric plexus and nerve fibers is more conspicuous in Hipk ${ }^{-1-}$ mutants at P35 than at P0, especially in the colon. Scale bar: (in $\left.\boldsymbol{L}\right) \mathbf{G}-\boldsymbol{L}, 200 \mu \mathrm{m}$.

bowel. The decrease in dopaminergic neurons also appeared to be more severe toward the caudal regions of the gut (Fig. 4O,P).

\section{Reduced TrkC-expressing neurons in the distal gut of Hipk $2^{-/-}$mutants}

We have previously reported that dopaminergic neurons constitute a subset of the TrkC-expressing (TrkC + ) neurons of the gut (Chalazonitis et al., 2008) and that BMP-2 and BMP-4 promote the development of TrkC+ neurons in cultures of enteric crestderived precursors (Chalazonitis et al., 2004). Consistent with this notion, NT-3 and BMP-4 promote differentiation of THexpressing neurons; moreover, the number of dopaminergic neurons is increased in transgenic mice that overexpress BMP-4 (NSE-BMP-4 mice) (Chalazonitis et al., 2004, 2008). Conversely, the number of TrkC+ neurons and DA neurons are reduced in the ENS of transgenic mice that overexpress the BMP antagonist Noggin (NSE-Nog mice) (Chalazonitis et al., 2004, 2008). Because there is a partial loss of ventral midbrain and enteric DA neurons in Hipk $2^{-1-}$ mutants (Fig. 4) (Zhang et al., 2007), we reasoned that the differentiation and maintenance of the TrkC+ neurons might also be affected in the Hipk2 $2^{-1-}$ mutant ENS. To test this hypothesis, we quantified TrkC+ neurons in the myenteric and submucosal plexuses using whole-mount preparations of duodenum, jejunum, and ileum of 12-week-old wild type and Hipk $2^{-I-}$ mutant mice (Fig. 5). Our results indicated that the packing density of TrkC + neurons per $\mathrm{mm}^{2}$ of plexus in the 


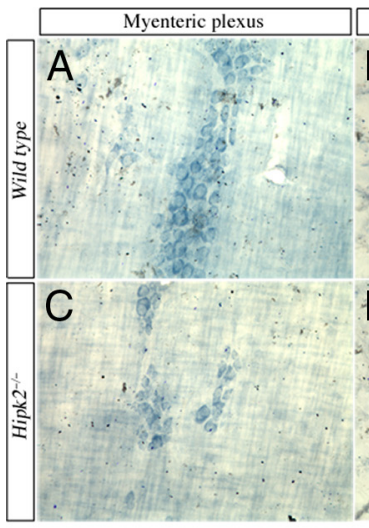

$\mathrm{E}$
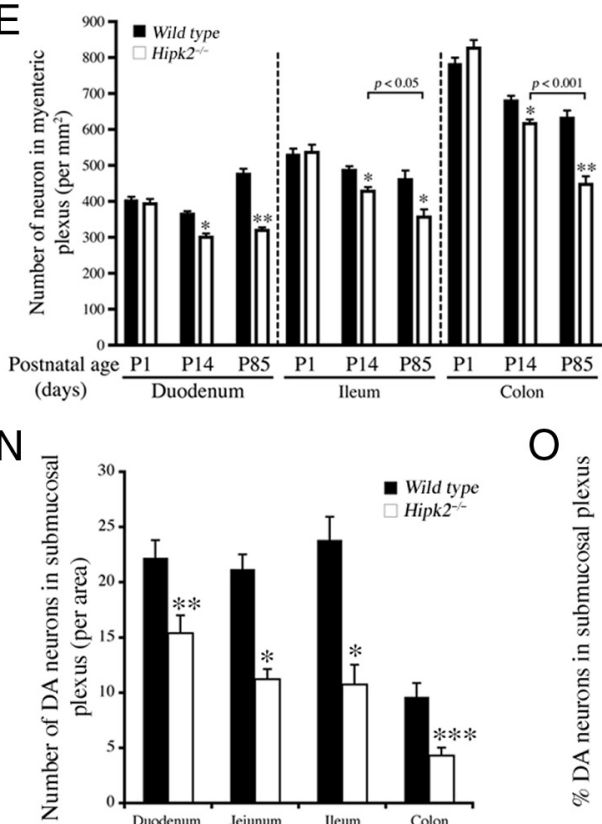

Submucosal plexus

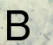

D

O

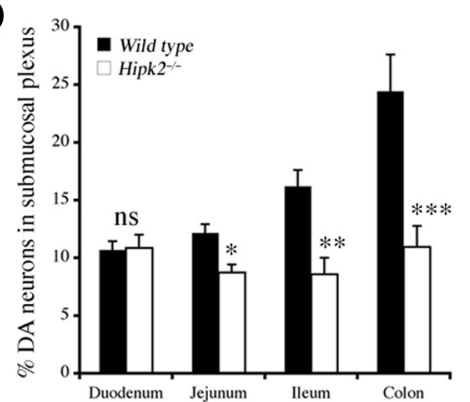

$\mathrm{F}$

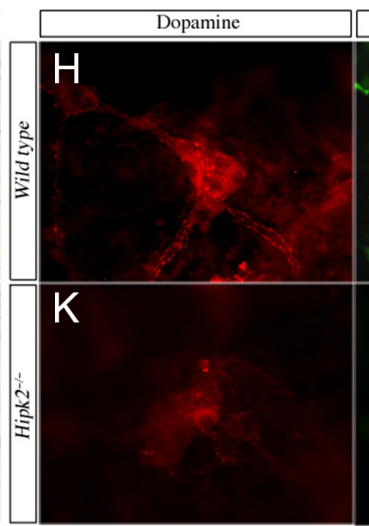

Tyrosine hydroxylase
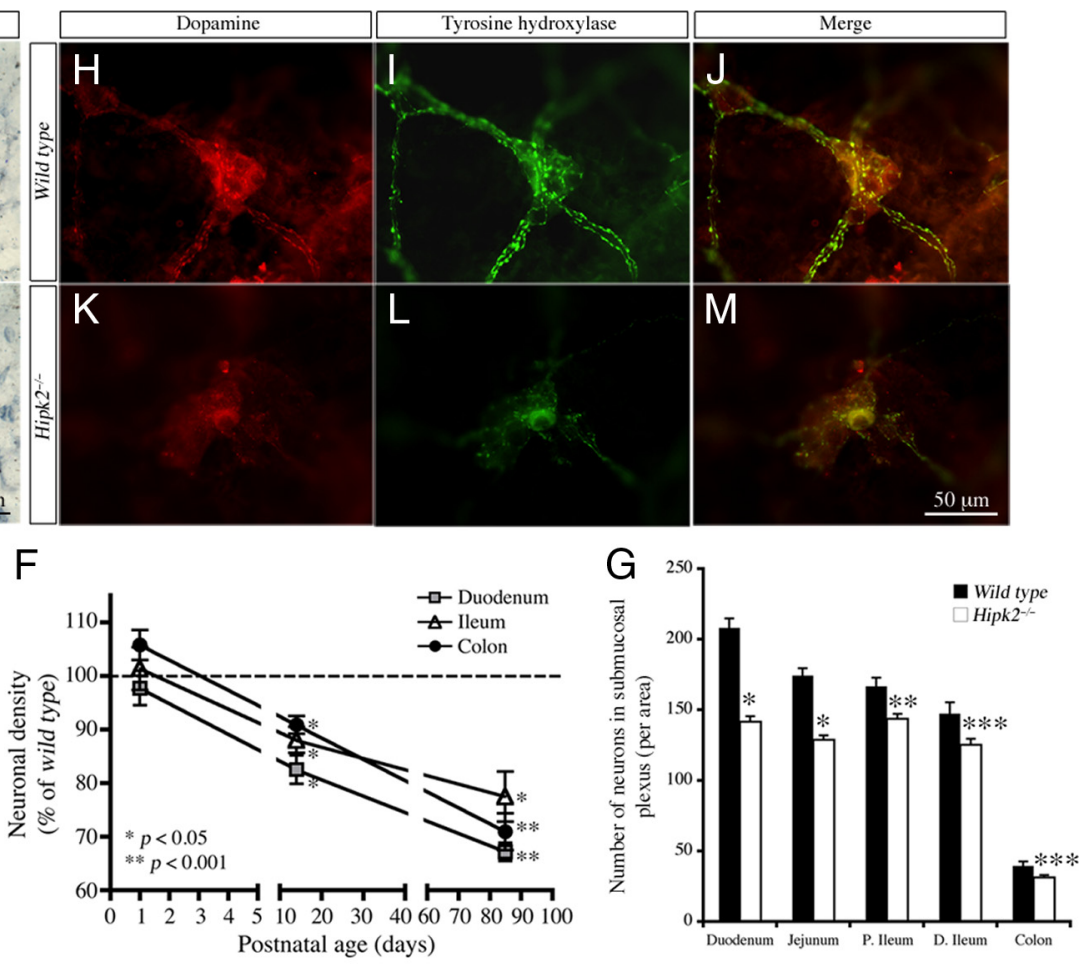

$\mathrm{P}$

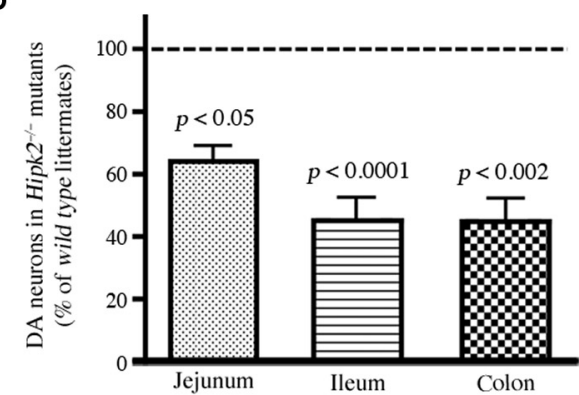

Figure 4. Loss of enteric neurons, including dopaminergic neurons, in Hipk $2^{-1-}$ mutant mice in postnatal life. $A-D$, Whole-mount cuprolinic blue staining of the myenteric and submucosal plexuses shows reduced neuronal density in adult Hipk $2^{-/-}$mutant mice. Scale bar: (in $\left.\boldsymbol{D}\right) \boldsymbol{A}-\boldsymbol{D}, 100 \mu \mathrm{m} . \boldsymbol{E}, \boldsymbol{F}$, Age-dependent reduction of neuron in the myenteric plexus of Hipk2 ${ }^{-/-}$mutants. At P1 there is no detectable neuron loss per unit area in the myenteric plexus of Hipk2 ${ }^{-1-}$ mutants. However, a progressive loss of neurons is present from P14 to P85 (or 12 weeks old) in the myenteric plexus of Hipk2 ${ }^{-1-}$ mutants. The age-dependent reduction of neurons in the myenteric plexus of Hipk2 ${ }^{-1-}$ mutants is demonstrated either as neuronal density per unit area $(\boldsymbol{E})$ or as a percentage of wild type neurons $(\boldsymbol{F})$. G, Similar to the myenteric plexus, reduction of neuronal density is also detected in the submucosal plexus of 12 -week-0ld (P84) Hipk2 ${ }^{-/-}$mutants. $\boldsymbol{H}-\boldsymbol{M}$, Dopaminergic neurons in the ENS are identified using whole-mount preparation of double immunofluorescence of dopamine (red) and tyrosine hydroxylase (green) in the submucosal plexus in wild type $(\boldsymbol{H}-\boldsymbol{J})$ and Hipk $2^{-/-}$mutant mice $(\boldsymbol{K}-\boldsymbol{M})$. Note that dopaminergic neurons are rarefied in Hipk $2^{-/-}$mutants compared to those in wild type littermates. Scale bar: (in $\left.\boldsymbol{M}\right) \boldsymbol{H}-\boldsymbol{M}, 50 \mu \mathrm{m}$. $\boldsymbol{N}$, Quantification of dopaminergic neuron density in duodenum, jejunum, ileum, and colon reveals a consistent reduction in dopaminergic neuron density in all regions. $\mathbf{0}, \boldsymbol{P}$, Even after the dopaminergic neuron density is normalized to the proportions of total number of neurons in submucosal plexus $(\boldsymbol{O})$ or as the percentage of wild type littermates $(\boldsymbol{P})$, there is still significant decrease in jejunum, ileum, and colon. At least three animals are counted for each genotype at P84, and the total numbers of measurement for each region of the gut are listed as follows: duodenum (wild type, $n=36$; Hipk2 ${ }^{-1-}, n=42$ ); jejunum (wild type, $n=36$; Hipk2 ${ }^{-1-}, n=23$ ); ileum ( $n=30$ for each genotype); and colon ( $n=13$ for each genotype). Student's $t$ test; ${ }^{*} p<0.05,{ }^{* *} p<$ $0.01,{ }^{* * *} p<0.005$, and ns (not significant).

Hipk $2^{-1-}$ mutants was significantly less than that in wild type mice in the myenteric plexus of jejunum $[59 \pm 4(n=21)$ compared to $74 \pm 3$ in wild type $(n=22), p<0.005]$ and ileum $[44 \pm$ $3(n=12)$ compared to $78 \pm 5$ in wild type $(n=18), p<0.0001]$. The decrease in the TrkC+ neuron packing density was also detected in the submucosal plexus of Hipk2 ${ }^{-1-}$ mice in the ileum $[21 \pm 1(n=21)$ compared to $37 \pm 2$ in wild type $(n=26), p<$ $0.0001]$ but not in the duodenum-jejunum $[44 \pm 2(n=36)$ compared to wild type $46 \pm 2(n=29)$ ] (Fig. 5A,B). Consistent with the reduction in $\mathrm{TrkC}+$ neuron packing density, TrkC+ neurons (expressed as percentage of wild type) in the myenteric plexus was reduced in duodenum-jejunum and even more in the ileum, while in the submucosal plexus the decrease was significant only in the ileum (Fig. $5 C$ ). These results were consistent with the proximodistal gradient in the severity of the reduction of enteric dopaminergic neurons (Fig. 4) and support the notion that, similar to its essential role for dopaminergic neurons, HIPK2 is required for the maintenance of TrkC + neurons in the ENS.

\section{Loss of HIPK2 increases BMP signaling in enteric neurons}

We have previously demonstrated that BMP-2 and BMP-4 regulate the development of enteric neurons, including that of the dopaminergic subset, enteric gangliogenesis, and enteric glia (Chalazonitis et al., 2004, 2008; Faure et al., 2007). Since BMP-2 and BMP-4 are members of the TGF $\beta$ superfamily and HIPK2 has been shown to interact physically with receptor-regulated Smads, including Smad1, Smad2, and Smad3, and modulate 

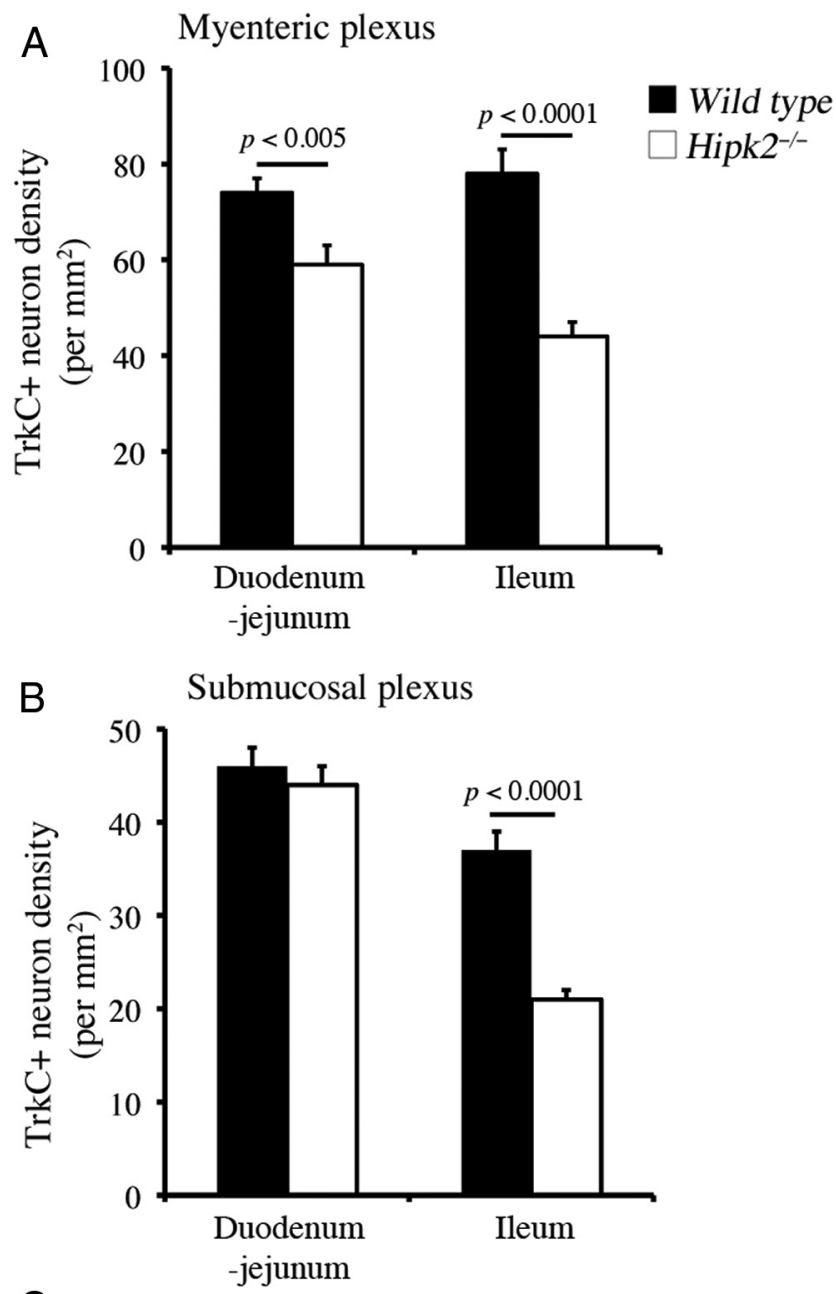
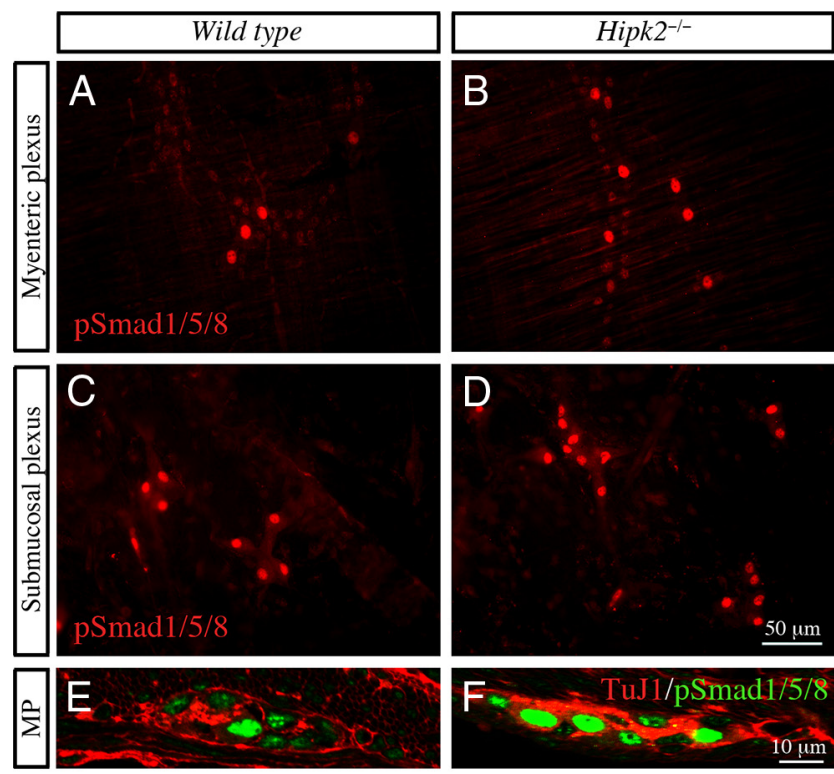

G Myenteric plexus

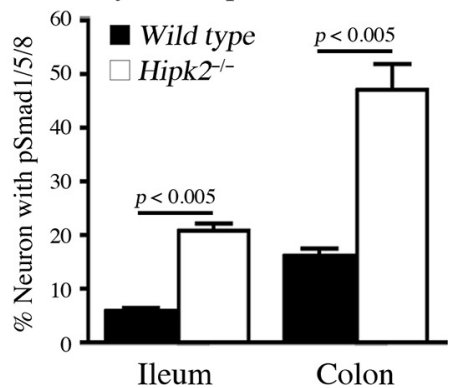

Submucosal plexus

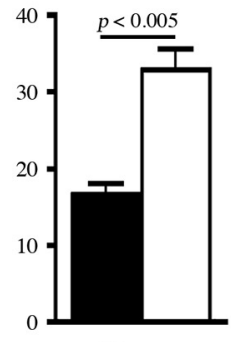

Ileum

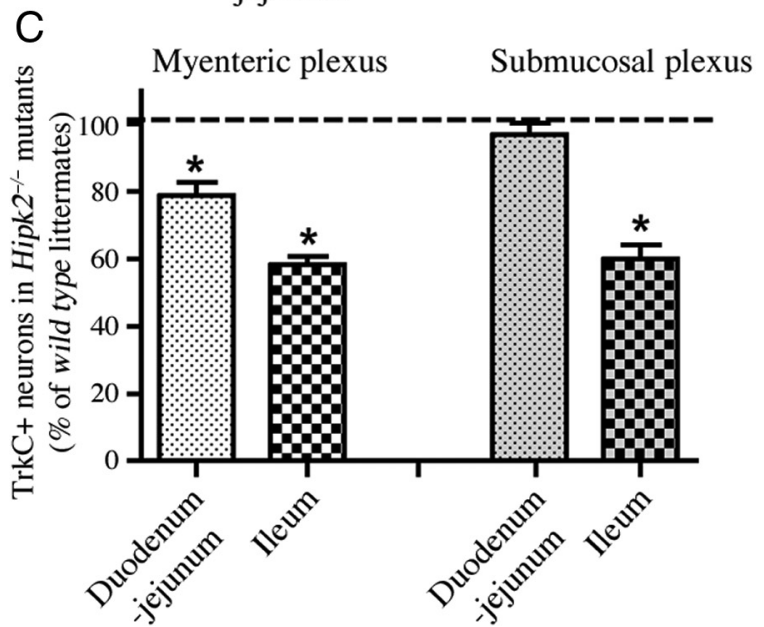

Figure 5. Hipk2 ${ }^{-1-}$ mutant mice show reduction in the TrkC + neurons in the myenteric and the submucosal plexus in ileum. $\boldsymbol{A}, \boldsymbol{B}$, Whole-mount immunohistochemistry highlights the reduction of TrkC + neurons in the myenteric plexus in duodenum-jejunum and ileum $(\boldsymbol{A})$. Reduction in TrkC + neuron is also detected in the submucosal plexus of Hipk $2^{-1-}$ mutants $(\boldsymbol{B})$, but not in duodenum-jejunum. $\boldsymbol{C}$, The density of Trk $\boldsymbol{C}+$ neurons in the Hipk2 $2^{-I-}$ mutant myenteric plexus, expressed as a percentage of wild type littermate control, shows a more significant reduction in the ileum $(n=15)$ than in the duodenum-jejunum $(n=21, p<0.0001)$. In contrast, the reduction of TrkC + neurons in the submucosal plexus is significant only in ileum $(n=21)$. Asterisk $\left(^{*}\right)$ indicates significant difference from wild type; Student's $t$ test, $p<0.0001$.
Figure 6. Loss of HIPK2 leads to increased expression of $\mathrm{pSmad1/5/8}$ in enteric neurons in myenteric and submucosal plexuses. $\boldsymbol{A}-\boldsymbol{F}$, Whole-mount immunostaining reveals a significant increase in pSmad1/5/8-positive neurons in the myenteric plexus $(\boldsymbol{A}, \boldsymbol{B})$ and submucosal plexus $(\boldsymbol{C}, \boldsymbol{D})$ in the ileum and colon in 12-week-old (P84) Hipk2 ${ }^{-1-}$ mutant mice compared to wild type. Note that the intensity of $\mathrm{pSmad} 1 / 5 / 8$ immunostaining varies among the enteric neurons. To be consistent, both the intensely and modestly positive enteric neurons are counted Scale bar: (in $\boldsymbol{D}) \boldsymbol{A}-\boldsymbol{D}, 50 \mu \mathrm{m} . \boldsymbol{E}, \boldsymbol{F}$, Histology sections demonstrate the colocalization of nuclear pSmad1/5/8 (green fluorescence) and neuronal marker, TuJ1 (red fluorescence), in the myenteric plexus in wild type and Hipk $2^{-1-}$ mutants. Scale bar: (in $\left.\boldsymbol{F}\right) \boldsymbol{E}, \boldsymbol{F}, 10 \mu \mathrm{m}$. $\boldsymbol{G}$, In the wild type myenteric plexus, $5.97 \pm 0.54 \%(n=30)$ and $16.2 \pm 1.4 \%(n=5)$ of total neurons show positive staining for $p S m a d 1 / 5 / 8$ in ileum and colon, respectively. In contrast, $20.8 \pm 1.4 \%$ and $47.2 \pm 4.7 \%$ of neurons are positive for $\mathrm{pSmad} 1 / 5 / 8$ in the ileum and colon of Hipk $2^{-1-}$ mutants, respectively. A similar increase in $\mathrm{pSmad1/5/8}$ is also detected in the submucosal plexus in the ileum of Hipk2 ${ }^{-1-}$ mutants, $32.8 \pm 2.7 \%(n=26)$ compared to $16.7 \pm 1.3 \%$ ( $n=35$ ) in wild type. Student's $t$ test, $n=5$ for each genotype, and $p<0.005$. Note that the increased proportion of neurons with $\mathrm{pSmad} 1 / 5 / 8$ immunostaining is significantly higher $(p<$ 0.005 ) in the colon than in the ileum of Hipk $2^{-1-}$ mice.

Smad-dependent gene expression (Harada et al., 2003; Zhang et al., 2007), we postulated that loss of HIPK2 might influence BMP signaling. To test this hypothesis, we characterized BMP signaling in enteric neurons by examining the expression of phosphorylated Smad1/5/8 (pSmad1/5/8) in enteric neurons in the myenteric and submucosal plexuses of wild type and Hipk2 ${ }^{-/-}$mutants. Whereas no difference in $\mathrm{pSmad} 1 / 5 / 8$ staining was found in the enteric neurons of wild type and Hipk2 ${ }^{-1-}$ mutants at P0, there were more neurons with positive nuclear $\mathrm{pSmad} 1 / 5 / 8$ staining in the myenteric plexus of the ileum and colon in 12-week-old Hipk2 ${ }^{-1-}$ mutants than there were in wild type littermates (Fig. $6 A$ vs $B$ and $E$ vs $F$, and data not shown). When normalized to the total number of neurons in the corresponding region of the gut, a much higher percentage of 

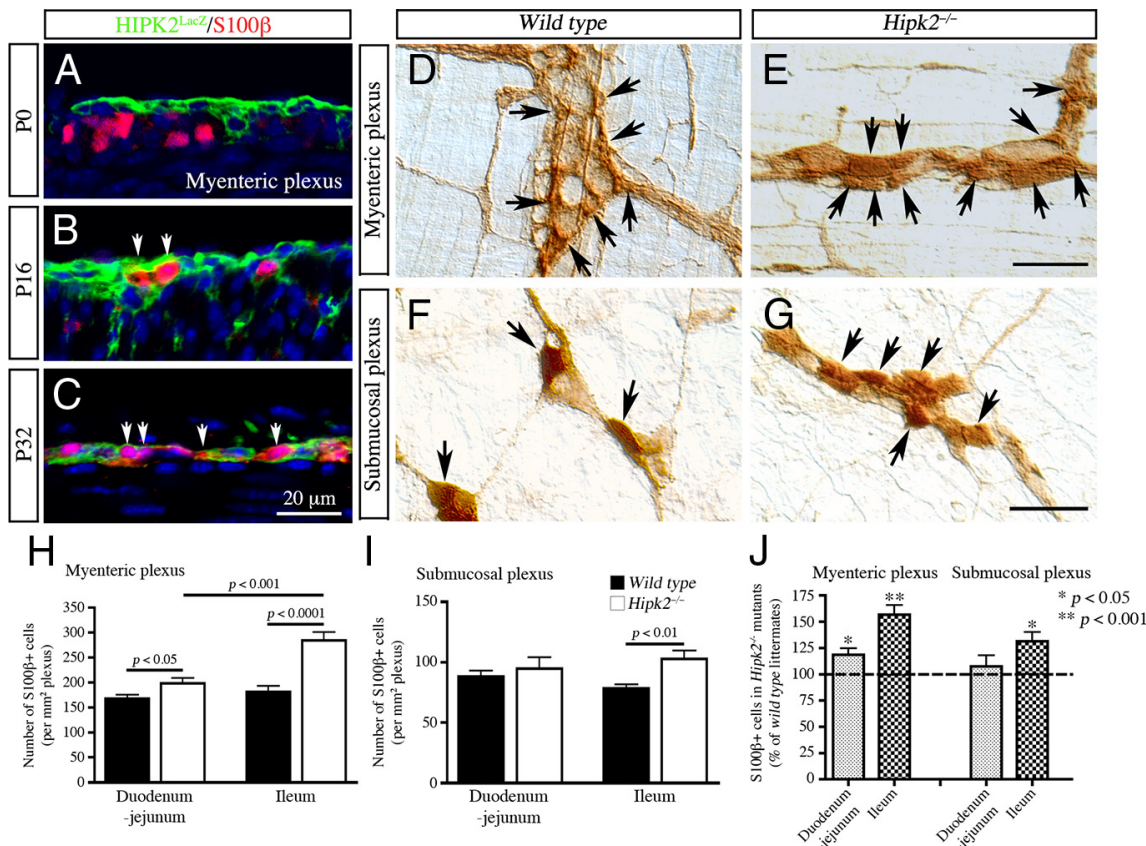

Figure 7. Hipk $2^{-1-}$ mutant mice show increase in glia density in the myenteric and the submucosal plexus. $\boldsymbol{A}-\boldsymbol{C}$, Confocal images showing expression of HIPK2 ${ }^{\text {LacZ }}$ (detected by anti- $\beta$-galactosidase antibody) in glia (detected by anti-S100 $\beta$ antibody). At P0 there is very limited expression of HIPK2 in the enteric glia $(\boldsymbol{A})$. However, the expression of HIPK2 ${ }^{\text {LacZ }}$ shows a progressive increase in the glia at P16 (B) and P32 (C) (arrows). Scale bar: (in C) $\boldsymbol{A}-\boldsymbol{C}, 20 \mu \mathrm{m}$. D-G. Whole-mount immunostaining for $5100 \beta$ shows increased enteric glia in the myenteric and submucosal plexuses of 12 -week-old Hipk2 ${ }^{-/-}$mutants compared to their wild type littermates ( $n=3$, each). The images are captured using a $40 \times$ objective under differential interference contrast microscopy that allows better delineation of glial and neuronal cell bodies. Note that the brown immunoreactivity of the glia cell bodies and their small nuclei (arrows) delineate the ganglia and that the glia processes wrap around the light lacuna of mostly larger neuronal cell bodies and nuclei. Scale bars: (in $\boldsymbol{E}, \mathbf{G}) \boldsymbol{D}-\mathbf{G}, 50 \mu \mathrm{m}$. $\boldsymbol{H}$, Glia density (per $\mathrm{mm}^{2}$ of plexus) in the myenteric plexus is increased in Hipk $2^{-1-}$ mutant mice in both duodenum-jejunum and ileum but is significantly higher in ileum. I, Glia density in the submucosal plexus of Hipk $2^{-1-}$ mutants is significantly increased only in the ileum. $J$, Glia density normalized as percentage of wild type corroborates the data expressed per $\mathrm{mm}^{2}$ of plexus.

neurons in the myenteric plexus of the ileum in Hipk $2^{-1-}$ mice $(20.8 \pm 1.3 \%)$ showed positive pSmad1/5/8 staining than that in wild type mice $(5.97 \pm 0.5 \%$ ) (Fig. $6 G$, left). pSmad $1 / 5 / 8$ staining was also greater in submucosal plexus neurons in Hipk2 ${ }^{-1-}$ mice $(32.8 \pm 2.7 \%)$ than in wild type animals $(16.7 \pm 1.3 \%)$ (Fig. $6 C$ vs $D$ and $G$, right, $p<0.005)$. A similar increase in $\mathrm{pSmad} 1 / 5 / 8$-positive neurons was also detected in the myenteric plexus of the colon in Hipk $2^{-l-}$ mutants $(47.2 \pm 4.7 \%)$ compared to the wild type (16.2 \pm $1.4 \%$ ) (Fig. $6 \mathrm{G}$ ). In contrast to the higher percentage of neurons with pSmad1/5/8 immunoreactivity, we found no detectable increase in pSmad2 staining in neurons of Hipk $2^{-l-}$ mutants (data not shown). The inverse relationship of more severe neuronal loss and a higher percentage of neurons with $\mathrm{pSmad} 1 / 5 / 8$ immunoreactivity in the ileum and colon of Hipk $2^{-/-}$mutants suggest that an aberrant increase in BMP signaling in enteric neurons may have contributed to the observed phenotype.

Increased glial density in the ENS of Hipk2 ${ }^{-1-}$ mutant mice In addition to their roles in enteric neuron development, BMP-2 and BMP-4 can also regulate gliogenesis in the ENS. Mice overexpressing BMP-4 (NSE-BMP-4) showed increased glial density and glia-to-neuron ratio (Chalazonitis et al., 2011). Because loss of HIPK2 leads to a significant increase in BMP signaling, we reasoned that the glial development in the ENS of Hipk $2^{-1-}$ mutants might be perturbed. To test this, we first determined whether HIPK2 is expressed in the enteric glia. Using antibodies that detected $\beta$-galactosidase (for HIPK2 ${ }^{\text {LacZ }}$ ) and S100 $\beta$ (for glia), we found that
HIPK2 was present in very few glia at $\mathrm{P} 0$ but showed a progressive increase from $\mathrm{P} 16$ to P32 (Fig. 7A-C). Remarkably, the density of glia in the myenteric plexus of adult (P32) Hipk $2^{-1-}$ mice (expressed per $\mathrm{mm}^{2}$ of plexus) was significantly increased in the duodenum $[198 \pm 10.4(n=15)$ in Hipk $2^{-1-}$ mutants compared to $167 \pm 7.8$ $(n=12)$ in wild type, $p<0.05]$ and in ileum $\left[284.3 \pm 16.5(n=12)\right.$ in Hipk2 ${ }^{-1-}$ mutants compared to $181.3 \pm 11.6(n=12)$ in wild type] (Fig. $7 D, E, H$ ). A similar increase in glial density was also detected in the submucosal plexus in the ileum of Hipk $2^{-1-}$ mice $[103 \pm 7(n=12)$ compared to $78.2 \pm$ $3.4(n=12)$ in wild type], but not in the duodenum (Fig. 7F, G,I). When normalized as percentage of wild type littermates, the glial density in Hipk $2^{-1-}$ mutants continued to show significant increases in the myenteric plexus of duodenum (118.4 \pm $6 \%)$ and ileum $(156.8 \pm 9 \%)$ and in the submucosal plexus of the ileum (131.4 \pm $8 \%$ ) (Fig. 7J). Thus, similar to the decrease in neuronal density, the increase in glial density in the ENS of Hipk2 ${ }^{-1-}$ mutants also showed a proximal-to-distal gradient.

\section{Increased autophagy, but not apoptosis, occurs in enteric neurons of Hipk2 ${ }^{-1-}$ mutants}

Our previous results have shown that loss of ventral midbrain DA neurons in Hipk $2^{-1-}$ mutants is due to increased apoptosis during the period of programmed cell death (Zhang et al., 2007). As is true of ventral midbrain DA neurons, the development of enteric neurons is also a long process that extends from the fetal period to postnatal life (Pham et al., 1991; Liu et al., 2009). It is unclear, however, whether enteric neurons, including those with a dopaminergic phenotype, exhibit programmed cell death during development (Gianino et al., 2003). To determine whether loss of enteric neurons in Hipk $2^{-1-}$ mutants was due to an increase in neuronal death, we used TUNEL assays in combination with the demonstration of $\beta 3$-tubulin immunoreactivity to quantify the number of neurons undergoing apoptosis. Because loss of enteric neurons in Hipk2 ${ }^{-1-}$ mutants does not become significant until the second postnatal week, we focused our analyses on enteric neurons at P7 and P14 mice. In contrast to the ventral midbrain dopaminergic neurons, apoptosis in the myenteric or submucosal plexus of Hipk2 ${ }^{-1-}$ mutants was not significantly different from that of WT mice at either age (data not shown). These observations suggest that the loss of neurons in Hipk2 ${ }^{-1-}$ mutants is not due to an increase in apoptosis.

To determine whether the loss of HIPK2 influenced maturation of enteric neurons in postnatal life, we used electron microscopy (EM) to examine the ENS of Hipk2 ${ }^{-1-}$ mutants. At P16, there was a significant increase in lysosomes and autophagosomes in the axons and cell bodies of neurons in Hipk2 $2^{-l-} \mathrm{mu}-$ tants (Fig. $8 B, D$ ). There were also many swollen axons that contained abnormal mitochondria and cytoplasmic vacuoles in the distal ileum. In contrast, axons in the wild type myenteric plexus were organized in tight bundles, and only very few small 
autophagosomes were noticed in axons or in cytoplasm (Fig. $8 \mathrm{~A}, \mathrm{C}$ ). Interestingly, as in the more severe neuronal phenotype in the distal parts of gut, there was a progressive increase in neurons with autophagosomes in the ileum and colon (Fig. 8E). Consistent with the increase in autophagosomes, there was a significant increase in the intensity of microtubule-associated protein-1 light chain 3 (LC3) immunofluorescence, an indicator of autophagy activity, at $\mathrm{P} 7$ and $\mathrm{P} 17$ in myenteric neurons of the colon in Hipk2 $2^{-l-}$ mutants (Fig. $8 F, G)$.

\section{Reduced intraganglionic synapses in} myenteric plexuses of Hipk2 $2^{-/-}$mice Myenteric neurons are known to form intraganglionic synapses and elaborate microcircuits that enable the ENS to manifest integrated neuronal activity and independently control the behavior of the bowel. The phenotype in Hipk2 $2^{-1-}$ mutants, including abnormal GITT, reduced density of neural connectives, and decreased numbers of neurons, suggested that the loss of HIPK2 might also affect synapse formation in the myenteric plexus. To test this hypothesis, we characterized the development of intraganglionic synapses in the intestines of Hipk $2^{-1-}$ mice. Whereas the expression of synaptic markers, such as SNAP-25, synaptotagmin, and PSD-95, showed similar robust staining in neurons in wild type submucosal and myenteric plexuses, these markers were substantially reduced in the intestines of adult Hipk2 $2^{-1-}$ mutants (Fig. $9 A-C$ vs $D-F$ ). To characterize further the cause of the reduced expression of synaptic markers in Hipk $2^{-1-}$ mutants, we employed EM to examine synaptic growth at various postnatal stages. As early as P0, discrete synaptic structures were found on enteric neurons in wild type and Hipk $2^{-1-}$ mice; these structures, which contained synaptic vesicles, synaptic clefts, and postsynaptic density, were observed in duodenum, ileum, and proximal colon (Fig. 9G-J). Interestingly, the density of synapses in the myenteric plexus increased progressively in postnatal life from P0 to P36, indicating that enteric neurons continue to mature postnatally for a considerable period of time (Fig. $9 \mathrm{~K}$ and data not shown). Compared to $\mathrm{P} 0$, the number of synapses in P36 wild type duodenum, ileum, and colon increased by 2-, 2.3- and 2.5-fold, respectively (Fig. $9 \mathrm{~K})$. In contrast, synapses in the myenteric plexus of Hipk $2^{-/-}$mice virtually failed to increase from P0 to P36 (Fig. $9 K)$. Importantly, whereas the density of synapses in Hipk $2^{-1-}$ mutants was not significantly reduced in duodenum and ileum, the synaptic density was reduced in the proximal colon at $\mathrm{P} 0(p=0.0068, n=4$, Fig. $9 K)$. The few synapses that were identified in the myenteric plexus of Hipk2 ${ }^{-1-}$ mice at P36 appeared poorly organized and lacked discrete synaptic structures, such as synaptic clefts and postsynaptic densities (Fig. 9H,J).Together, the EM analyses of postnatal enteric neurons support the idea that loss of HIPK2 leads to a prominent increase in autophagosomes and an arrest in synaptogenesis and maturation.
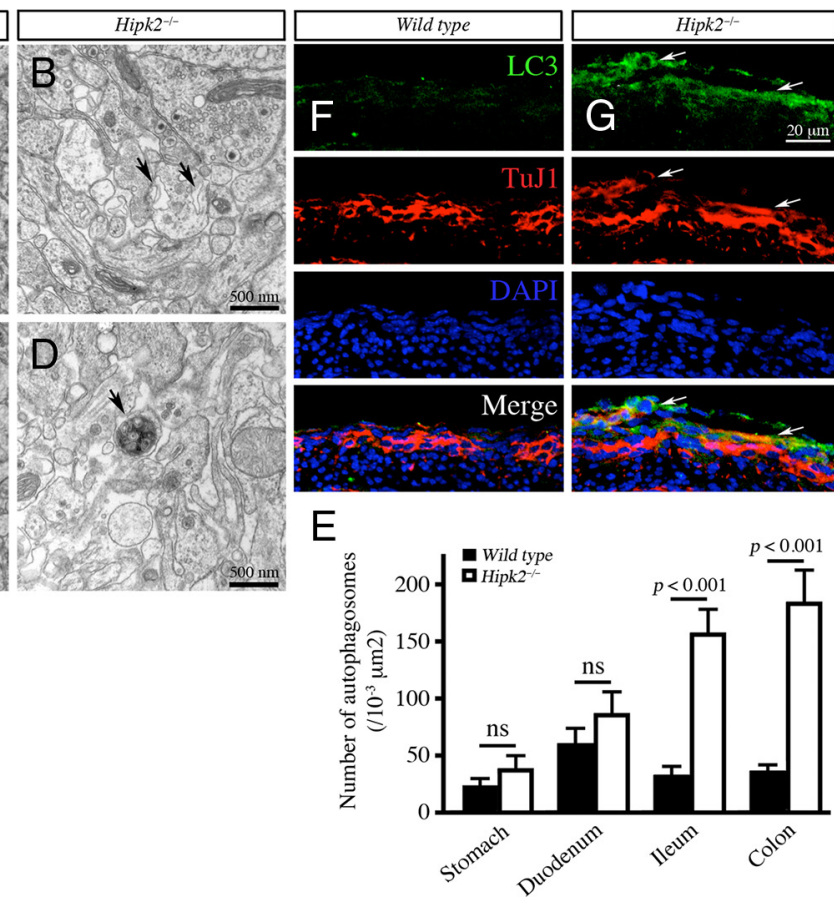

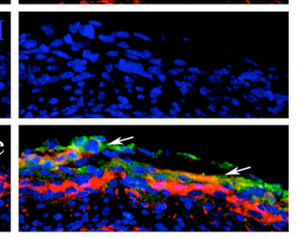

- mutants. $\boldsymbol{A}-\boldsymbol{D}$, Electron microscopic analyses show an $-D$, Electron microscopic analyses show an
mutants atP16 (B, D, arrows). In contrast, axons in neurons in the myenteric plexus of wild type mice show compact bundles and no evidence of autophagosomes $(\boldsymbol{A}, \boldsymbol{C})$. Scale
bars: (in $\boldsymbol{B}, \boldsymbol{D}) \boldsymbol{A}-\boldsymbol{D}, 500 \mu \mathrm{m}$. $\boldsymbol{E}$, Quantification of the number of autophagosomes (per $10^{-3} \mu \mathrm{m}^{2}$ surface area) in stomach ( $n=$ 31 in wild type and $n=15$ in Hipk2 ${ }^{-1-}$ mutants), duodenum ( $n=21$ in wild type and $n=16$ in Hipk2 ${ }^{-1-}$ mutants), ileum ( $n=$ 列 Whereas no significant difference in autophagosome is detected in stomach and duodenum, there are significant increases in the mutants. $\boldsymbol{F}, \boldsymbol{G}$, Consistent with the EM findings, the enteric neurons in the myenteric plexus of a diffuse increase in $L(3$ immunofluorescence signals (arrows). Scale bar: (in $\boldsymbol{G}) \boldsymbol{F}, \boldsymbol{G}, 20 \mu \mathrm{m}$.

\section{Discussion}

Our results underscore the important role of HIPK2 in the postnatal development of enteric neurons and glia. Loss of HIPK2 has no effect on the prenatal development of enteric neurons or its progenitors but leads to a progressive loss of enteric neurons and an arrest in synaptic maturation in postnatal life. Although there is no detectable reduction in enteric neurons at P0, Hipk2 ${ }^{-1-}$ mutants show distended stomachs and small intestines at perinatal stages (Fig. 1). These phenotypes are not caused by congenital malformations such as tracheoesophageal fistula, which may have interfered with feeding. Instead, two factors may potentially contribute to the gut motility problems in Hipk $2^{-1-}$ mutants at P0. First, there is a significant reduction in the synaptic density in the proximal colon of Hipk2 $2^{-1-}$ mutants at P0 (Fig. 9). In addition, modest reductions of synaptic density are also detected in the duodenum and ileum in Hipk $2^{-1-}$ mutants at P0 (Fig. 9). These synaptic defects may lead to functional impairments in the gut motility at perinatal stages and may persist into adulthood. Second, it is possible that defects in ventral midbrain DA neurons in Hipk $2^{-1-}$ mutants at perinatal stages may interfere with their ability to feed (Zhang et al., 2007).

The significant loss of dopaminergic neurons in the ENS of Hipk $2^{-1-}$ mutants in postnatal stages is reminiscent of the dopaminergic neuron phenotype in the substantia nigra pars compacta (SNpc) and the ventral tegmental area (VTA) (Zhang et al., 2007). There are, however, several distinct differences in the Hipk $2^{-1-}$ mutant dopaminergic phenotype in the ENS and ventral midbrain (vMB). For example, the onset of dopaminergic neuron deficits in SNpc and VTA in Hipk $2^{-1-}$ mutants coincides 


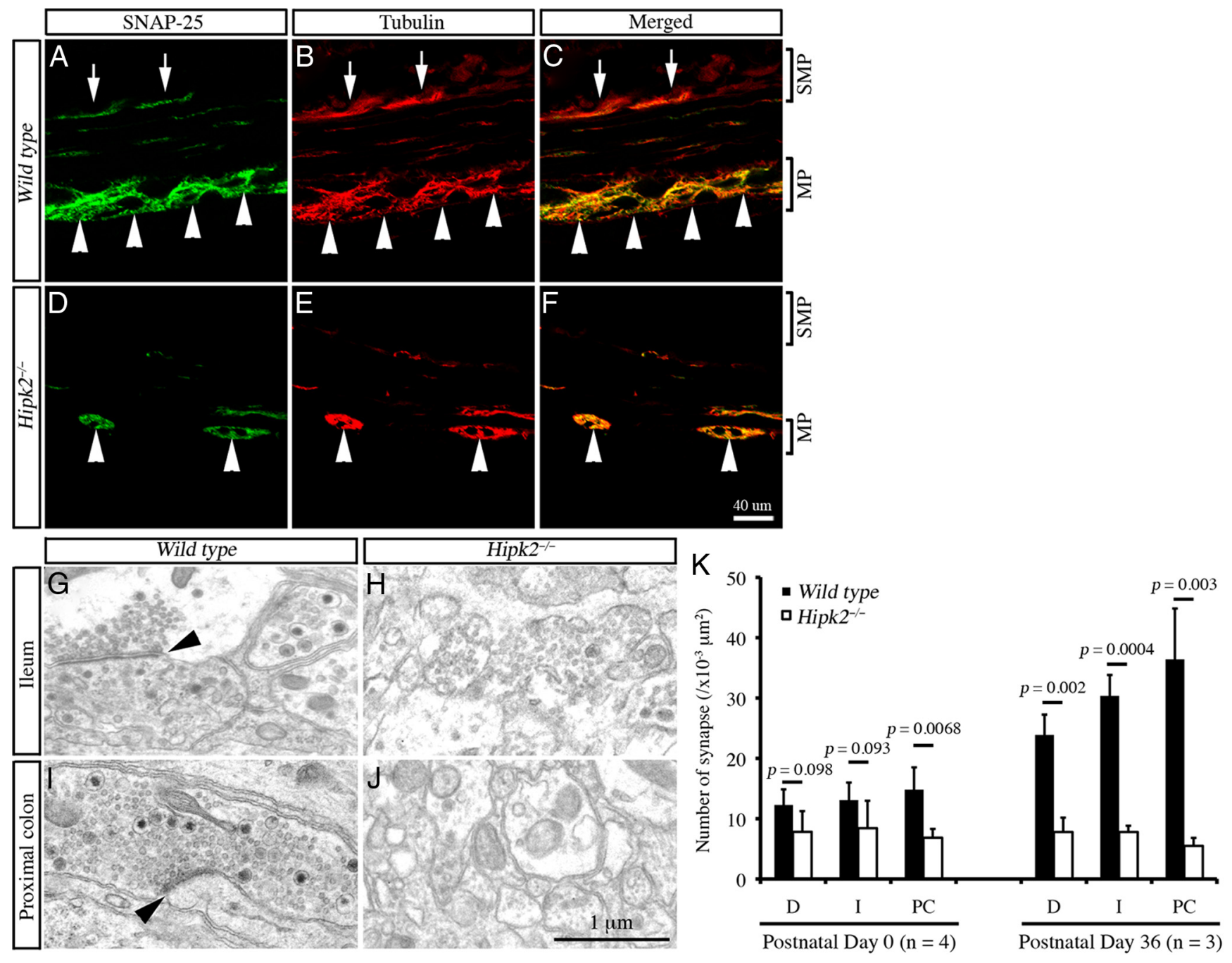

Figure 9. Reduced intraganglionic synapses in the myenteric plexus of Hipk $2^{-1-}$ mutants. $\boldsymbol{A}-\boldsymbol{F}$, Immunofluorescent images of synaptic protein, SNAP-25, show reduced signal intensity in the myenteric plexus (MP) and submucosal plexus (SMP) in colon of P36 Hipk2 ${ }^{-1-}$ mutants. Arrowheads indicate neurons in myenteric plexus, and arrows indicate neurons in the submucosal plexus. Scale bar: (in $\boldsymbol{F}) \boldsymbol{A}-\boldsymbol{F}, 40 \mu \mathrm{m} . \mathbf{G}-\boldsymbol{J}$, Representative EM images of the synapses in the myenteric plexus in ileum and proximal colons in adult wild type $\left(\boldsymbol{G}\right.$ and $\boldsymbol{I}$, respectively) and $H_{i p k 2}{ }^{-1-}$ mutants $\left(\boldsymbol{H}\right.$ and $\boldsymbol{J}$, respectively). Whereas the synapses are well developed in the enteric neurons in wild type mice $(G, I)$, the enteric neurons of $H i p k 2^{-1-}$ mutants show reduced number of synapse in ileum and proximal colon $(\boldsymbol{H}, \boldsymbol{J})$. Scale bar: (in $J) \mathbf{G}-\boldsymbol{J}, 1 \mu \mathrm{m}$. $\boldsymbol{K}$, Quantification of the number of synapses per unit area $\left(\times 10^{-3} \mu \mathrm{m}^{3}\right.$ ) in duodenum (D), ileum (I), and proximal colon (PC) at P0 and P36 shows a significant increase in synaptic density in the ENS in postnatal life in wild type mice. In contrast, Hipk2 ${ }^{-1-}$ mutant mice show a modest reduction in synaptic density at PO with no further increase in synaptic density from P0 to P36.

with a significant increase in apoptotic neurons during the period of programmed cell death. Ventral midbrain dopaminergic neurons from Hipk $2^{-1-}$ mutants also fail to survive in the presence of TGF $\beta$, suggesting that HIPK2 is required to transmit the prosurvival signals from TGF $\beta$ in these neurons. Consistent with this notion, both in vitro and in vivo studies support the notion that TGF $\beta$ isoforms are prosurvival trophic factors for the midbrain dopaminergic neurons (Poulsen et al., 1994; Krieglstein et al., 2000; Zhang et al., 2007). In contrast, there is no detectable increase in apoptosis in the ENS of Hipk $2^{-1-}$ mutants, and the time course for the loss of enteric neurons in Hipk $2^{-1-}$ mutants follows a rather long postnatal interval. While the underlying mechanism of the neuronal deficit in Hipk2 ${ }^{-1-}$ mutants remains unclear, it is interesting to note that, unlike sympathetic and sensory neurons, deprivation of trophic factor support for enteric neurons does not seem to trigger conventional apoptotic cell death. In a previous study (Gianino et al., 2003), no increase in caspase- 3 immunoreactivity was observed in the enteric neurons at fetal, early postnatal, or adult stages in mice. Interestingly, loss of the GDNF receptor, GFR $\alpha 1$, in the ENS leads to unconventional cell death that is not associated with the activation of common cell death executors, such as caspase- 3 or caspase-7. Neuronal death induced by GDNF deprivation, furthermore, cannot be blocked by caspase inhibitors or Bax deficiency (Uesaka et al., 2007). The only documented example of apoptotic cell death that can be related to the ENS occurs in early vagal neural crest-derived cells, which are migrating progenitors en route to the bowel. Blocking cell death in migrating vagal crest-derived cells by using dominant negative caspase- 9 in developing chick embryos increases the number of enteric neurons (hyperganglionosis) in the proximal foregut (Wallace et al., 2009). Together, these results suggest that the manifestation of apoptosis in the ENS may be stage dependent and that loss of trophic factor support in postmitotic neurons may lead to removal of these neurons via unconventional degenerative processes (Enomoto, 2009).

Although TGF $\beta$ isoforms support the survival of vMB dopaminergic neurons, their roles in the survival and maturation of 
enteric neurons are not clear. In cultures of enteric neural crestderived cells, we have shown that low doses of BMP-2 or BMP-4 promote while high doses and prolonged exposure inhibit the development of enteric neurons. These results suggest that the effects of BMPs on enteric neuron development may be contextdependent (Chalazonitis et al., 2004). Consistent with this idea, transgenic mice that overexpress the BMP antagonist Noggin show an increased number of neurons that exit the cell cycle early but have a reduced number of neurons, such as the dopaminergic neurons, that exit the cell cycle late (Chalazonitis et al., 2004, 2008). It is important to note that higher than normal levels of pSmad $1 / 5 / 8$ are found in the remaining enteric neurons of Hipk $2^{-1-}$ mutants (Fig. 6); however, there is no detectable change in the pSmad2 level (A.A. Tang and Y. Shang, unpublished observations). While it is unclear how loss of HIPK2 leads to upregulation in pSmad1/5/8, we do know that HIPK2 is a transcriptional corepressor that can directly interact with Smad1 and suppress BMP-dependent reporter gene expression (Harada et al., 2003). Consistent with the results in Figure 6, pSmad1/5/8 is upregulated in mouse embryonic fibroblasts (MEFs) from Hipk $2^{-1-}$ mutants upon treatment with BMP. In contrast, when treated with $\mathrm{TGF} \beta$, Hipk2 ${ }^{-1-}$ MEFs show significantly less pSmad2 than wild type MEFs (Y. Shang, unpublished observations). It is thus possible that HIPK2 may be required to prevent excessive BMP signaling by suppressing the activation of Smad1/ 5/8. As a consequence, loss of HIPK2 results in a constitutive upregulation of the BMP signaling mechanism, which may influence neuronal survival and maturation and promotes gliogenesis in postnatal life.

Our observations indicate that the enteric neurons of Hipk $2^{-1-}$ mutants contain increased number of autophagosomes and exhibit synaptic growth arrests from P0 to P36 (Figs. 8, $9)$. One potential contributing factor to the synaptic deficit in Hipk $2^{-1-}$ mutants could be the marked upregulation of pSmad1/5/8 in Hipk2 $2^{-1-}$ enteric neurons (Fig. 6). In support of this hypothesis, several studies have indicated that the BMP ortholog, Glass bottom boat (Gbb), and the BMP type II receptor, Wishful thinking (Wit), in Drosophila promote the growth and synaptic homeostasis of the neuromuscular junction (Aberle et al., 2002; McCabe et al., 2003; Goold and Davis, 2007). TGF $\beta 1$, furthermore, can also regulate long-term synaptic potentiation, neuronal excitability, and synapsin distribution in Aplysia neurons (Zhang et al., 1997; Chin et al., 2002, 2006). It is possible that constitutive activation of the BMP signaling pathway in the absence of HIPK2 adversely influences synaptic growth and homeostasis in postnatal enteric neurons. Several recent studies indicate that, in addition to their roles in regulating synaptic growth, TGF $\beta$ and BMP signaling can influence the induction of autophagy in both normal and cancer cells (Kiyono et al., 2009; Suzuki et al., 2010; Xavier et al., 2010). Perturbations in BMP and/or TGF $\beta$ signaling pathways in the enteric neurons of Hipk $2^{-1-}$ mutants may thus have contributed to the increase in autophagy in these neurons. It is intriguing to note that the cellular and synaptic phenotype of Hipk2 $2^{-1-}$ mutants is more prominent in the ileum and colon than in the proximal intestine. While the exact cause of this phenotype is still unclear, it is possible that there is a rostrocaudal gradient in enteric expression of BMPs (Goldstein et al., 2005) similar to the dorsal-ventral gradient of BMPs that contributes to patterning of the forebrain and spinal cord (Liu and Niswander, 2005).

Finally, our analyses of the DA neuronal phenotype in Hipk $2^{-I-}$ mutants provide a potential insight into the pathogenesis of Parkinson's disease. It is well documented that patients with Parkinson's disease show manifestations of symptoms and Lewy body pathology in the ENS at the very early stage of disease progression, including reduced dopaminergic neuron numbers and dopamine level in the muscularis externa (Wakabayashi et al., 1990; Singaram et al., 1995). Expression in transgenic mice of mutated $\alpha$-synuclein, which is found in genetic forms of Parkinson's disease, leads to degenerative changes in the ENS, abnormal motility, and aggregates of $\alpha$-synuclein in enteric neurons (Kuo et al., 2010). The intestinal dysfunction of these mice is not dissimilar to that seen in Hipk2 $2^{-1-}$ mutants (Fig. 1). These observations lead to the hypothesis that the ENS plays an important role in the pathogenesis and pathophysiology of Parkinson's disease (Lebouvier et al., 2009). Despite these exciting observations, there is a persistent lack of evidence that the dopaminergic phenotype in Parkinson's patients or animal models can be causally related to a given signaling mechanism. The results from our current and previous studies indicate that the Hipk2 ${ }^{-1-}$ mutant mice provide an intriguing example where loss of HIPK2, a key signaling component in the TGF $\beta$ and BMP signaling pathways, leads to structural and functional impairments in dopaminergic neurons in vMB and the ENS (Zhang et al., 2007). Many prior studies, which support our results, have implicated TGF $\beta$ in the development, survival, and neuroprotection of dopaminergic neurons in a number of experimental paradigms (Roussa et al., 2009). Future studies are needed to determine whether components of the TGF $\beta$ or BMP signaling pathways can be used as therapeutic targets to prevent or treat congenital dysmotility syndromes or acquired conditions such as Parkinson's disease that compromise the function of the ENS.

\section{References}

Aberle H, Haghighi AP, Fetter RD, McCabe BD, Magalhães TR, Goodman CS (2002) wishful thinking encodes a BMP type II receptor that regulates synaptic growth in Drosophila. Neuron 33:545-558.

Blaugrund E, Pham TD, Tennyson VM, Lo L, Sommer L, Anderson DJ, Gershon MD (1996) Distinct subpopulations of enteric neuronal progenitors defined by time of development, sympathoadrenal lineage markers and Mash-1-dependence. Development 122:309-320.

Burns AJ, Pachnis V (2009) Development of the enteric nervous system: bringing together cells, signals and genes. Neurogastroenterol Motil 21:100-102.

Carai MA, Colombo G, Gessa GL, Yalamanchili R, Basavarajappa BS, Hungund BL (2006) Investigation on the relationship between cannabinoid $\mathrm{CB1}$ and opioid receptors in gastrointestinal motility in mice. Br J Pharmacol 148:1043-1050.

Chalazonitis A, D'Autréaux F, Guha U, Pham TD, Faure C, Chen JJ, Roman D, Kan L, Rothman TP, Kessler JA, Gershon MD (2004) Bone morphogenetic protein-2 and -4 limit the number of enteric neurons but promote development of a TrkC-expressing neurotrophin-3-dependent subset. J Neurosci 24:4266-4282.

Chalazonitis A, Pham TD, Li Z, Roman D, Guha U, Gomes W, Kan L, Kessler JA, Gershon MD (2008) Bone morphogenetic protein regulation of enteric neuronal phenotypic diversity: relationship to timing of cell cycle exit. J Comp Neurol 509:474-492.

Chalazonitis A, D’Autréaux F, Pham TD, Kessler JA, Gershon MD (2011) Bone morphogenetic proteins regulate enteric gliogenesis by modulating ErbB3 signaling. Dev Biol 350:64-79.

Chin J, Angers A, Cleary LJ, Eskin A, Byrne JH (2002) Transforming growth factor betal alters synapsin distribution and modulates synaptic depression in Aplysia. J Neurosci 22:RC220.

Chin J, Liu RY, Cleary LJ, Eskin A, Byrne JH (2006) TGF-betal-induced long-term changes in neuronal excitability in Aplysia sensory neurons depend on MAPK. J Neurophysiol 95:3286-3290.

Derynck R, Zhang YE (2003) Smad-dependent and Smad-independent pathways in TGF-beta family signalling. Nature 425:577-584.

Doxakis E, Huang EJ, Davies AM (2004) Homeodomain-interacting protein kinase-2 regulates apoptosis in developing sensory and sympathetic neurons. Curr Biol 14:1761-1765. 
Enomoto H (2009) Death comes early: apoptosis observed in ENS precursors. Neurogastroenterol Motil 21:684-687.

Enomoto H, Araki T, Jackman A, Heuckeroth RO, Snider WD, Johnson EM Jr, Milbrandt J (1998) GFR alpha1-deficient mice have deficits in the enteric nervous system and kidneys. Neuron 21:317-324.

Faure C, Chalazonitis A, Rhéaume C, Bouchard G, Sampathkumar SG, Yarema KJ, Gershon MD (2007) Gangliogenesis in the enteric nervous system: roles of the polysialylation of the neural cell adhesion molecule and its regulation by bone morphogenetic protein-4. Dev Dyn 236:44-59.

Flynn B, Bergner AJ, Turner KN, Young HM, Anderson RB (2007) Effect of Gdnf haploinsufficiency on rate of migration and number of enteric neural crest-derived cells. Dev Dyn 236:134-141.

Fu M, Vohra BP, Wind D, Heuckeroth RO (2006) BMP signaling regulates murine enteric nervous system precursor migration, neurite fasciculation, and patterning via altered Ncam1 polysialic acid addition. Dev Biol 299:137-150.

Furness JB (2006) The enteric nervous system. Oxford: Blackwell.

Gershon MD (2010) Developmental determinants of the independence and complexity of the enteric nervous system. Trends Neurosci 33:446-456.

Gianino S, Grider JR, Cresswell J, Enomoto H, Heuckeroth RO (2003) GDNF availability determines enteric neuron number by controlling precursor proliferation. Development 130:2187-2198.

Goldstein AM, Brewer KC, Doyle AM, Nagy N, Roberts DJ (2005) BMP signaling is necessary for neural crest cell migration and ganglion formation in the enteric nervous system. Mech Dev 122:821-833.

Goold CP, Davis GW (2007) The BMP ligand Gbb gates the expression of synaptic homeostasis independent of synaptic growth control. Neuron 56:109-123.

Harada J, Kokura K, Kanei-Ishii C, Nomura T, Khan MM, Kim Y, Ishii S (2003) Requirement of the co-repressor homeodomain-interacting protein kinase 2 for ski-mediated inhibition of bone morphogenetic proteininduced transcriptional activation. J Biol Chem 278:38998-39005.

Heinicke EA, Kiernan JA, Wijsman J (1987) Specific, selective, and complete staining of neurons of the myenteric plexus, using cuprolinic blue. J Neurosci Methods 21:45-54.

Karaosmanoglu T, Aygun B, Wade PR, Gershon MD (1996) Regional differences in the number of neurons in the myenteric plexus of the guinea pig small intestine and colon: an evaluation of markers used to count neurons. Anat Rec 244:470-480.

Kiyono K, Suzuki HI, Matsuyama H, Morishita Y, Komuro A, Kano MR, Sugimoto K, Miyazono K (2009) Autophagy is activated by TGF-beta and potentiates TGF-beta-mediated growth inhibition in human hepatocellular carcinoma cells. Cancer Res 69:8844-8852.

Krieglstein K, Richter S, Farkas L, Schuster N, Dünker N, Oppenheim RW, Unsicker K (2000) Reduction of endogenous transforming growth factors beta prevents ontogenetic neuron death. Nat Neurosci 3:1085-1090.

Kuo YM, Li Z, Jiao Y, Gaborit N, Pani AK, Orrison BM, Bruneau BG, Giasson BI, Smeyne RJ, Gershon MD, Nussbaum RL (2010) Extensive enteric nervous system abnormalities in mice transgenic for artificial chromosomes containing Parkinson disease-associated alpha-synuclein gene mutations precede central nervous system changes. Hum Mol Genet 19:1633-1650.

Lebouvier T, Chaumette T, Paillusson S, Duyckaerts C, Bruley des Varannes S, Neunlist M, Derkinderen P (2009) The second brain and Parkinson's disease. Eur J Neurosci 30:735-741.

Li ZS, Pham TD, Tamir H, Chen JJ, Gershon MD (2004) Enteric dopaminergic neurons: definition, developmental lineage, and effects of extrinsic denervation. J Neurosci 24:1330-1339.

LiZS, Schmauss C, Cuenca A, Ratcliffe E, Gershon MD (2006) Physiological modulation of intestinal motility by enteric dopaminergic neurons and the D2 receptor: analysis of dopamine receptor expression, location, development, and function in wild-type and knock-out mice. J Neurosci 26:2798-2807.

Liu A, Niswander LA (2005) Bone morphogenetic protein signalling and vertebrate nervous system development. Nat Rev Neurosci 6:945-954.
Liu MT, Kuan YH, Wang J, Hen R, Gershon MD (2009) 5-HT4 receptormediated neuroprotection and neurogenesis in the enteric nervous system of adult mice. J Neurosci 29:9683-9699.

Massagué J, Seoane J, Wotton D (2005) Smad transcription factors. Genes Dev 19:2783-2810.

McCabe BD, Marqués G, Haghighi AP, Fetter RD, Crotty ML, Haerry TE, Goodman CS, O'Connor MB (2003) The BMP homolog Gbb provides a retrograde signal that regulates synaptic growth at the Drosophila neuromuscular junction. Neuron 39:241-254.

Pham TD, Gershon MD, Rothman TP (1991) Time of origin of neurons in the murine enteric nervous system: sequence in relation to phenotype. J Comp Neurol 314:789-798.

Poulsen KT, Armanini MP, Klein RD, Hynes MA, Phillips HS, Rosenthal A (1994) TGF beta 2 and TGF beta 3 are potent survival factors for midbrain dopaminergic neurons. Neuron 13:1245-1252.

Roussa E, von Bohlen und Halbach O, Krieglstein K (2009) TGF-beta in dopamine neuron development, maintenance and neuroprotection. Adv Exp Med Biol 651:81-90.

Sang Q, Young HM (1998) The identification and chemical coding of cholinergic neurons in the small and large intestine of the mouse. Anat Rec 251:185-199.

Singaram C, Ashraf W, Gaumnitz EA, Torbey C, Sengupta A, Pfeiffer R, Quigley EM (1995) Dopaminergic defect of enteric nervous system in Parkinson's disease patients with chronic constipation. Lancet 346:861-864.

Suzuki HI, Kiyono K, Miyazono K (2010) Regulation of autophagy by transforming growth factor-beta (TGFbeta) signaling. Autophagy 6:645-647.

Uesaka T, Enomoto H (2010) Neural precursor death is central to the pathogenesis of intestinal aganglionosis in Ret hypomorphic mice. J Neurosci 30:5211-5218.

Uesaka T, Jain S, Yonemura S, Uchiyama Y, Milbrandt J, Enomoto H (2007) Conditional ablation of GFRalphal in postmigratory enteric neurons triggers unconventional neuronal death in the colon and causes a Hirschsprung's disease phenotype. Development 134:2171-2181.

Uesaka T, Nagashimada M, Yonemura S, Enomoto H (2008) Diminished Ret expression compromises neuronal survival in the colon and causes intestinal aganglionosis in mice. J Clin Invest 118:1890-1898.

Wakabayashi K, Takahashi H, Ohama E, Ikuta F (1990) Parkinson's disease: an immunohistochemical study of Lewy body-containing neurons in the enteric nervous system. Acta Neuropathol 79:581-583.

Wallace AS, Barlow AJ, Navaratne L, Delalande JM, Tauszig-Delamasure S, Corset V, Thapar N, Burns AJ (2009) Inhibition of cell death results in hyperganglionosis: implications for enteric nervous system development. Neurogastroenterol Motil 21:768-e49.

Wang H, Hughes I, Planer W, Parsadanian A, Grider JR, Vohra BP, KellerPeck C, Heuckeroth RO (2010) The timing and location of glial cell line-derived neurotrophic factor expression determine enteric nervous system structure and function. J Neurosci 30:1523-1538.

Wiggins AK, Wei G, Doxakis E, Wong C, Tang AA, Zang K, Luo EJ, Neve RL, Reichardt LF, Huang EJ (2004) Interaction of Brn3a and HIPK2 mediates transcriptional repression of sensory neuron survival. J Cell Biol 167:257-267.

Xavier S, Gilbert V, Rastaldi MP, Krick S, Kollins D, Reddy A, Bottinger E, Cohen CD, Schlondorff D (2010) BAMBI is expressed in endothelial cells and is regulated by lysosomal/autolysosomal degradation. PLoS One 5:e12995.

Zhang F, Endo S, Cleary LJ, Eskin A, Byrne JH (1997) Role of transforming growth factor-beta in long-term synaptic facilitation in Aplysia. Science 275:1318-1320.

Zhang J, Pho V, Bonasera SJ, Holtzman J, Tang AT, Hellmuth J, Tang S, Janak PH, Tecott LH, Huang EJ (2007) Essential function of HIPK2 in TGFbeta-dependent survival of midbrain dopamine neurons. Nat Neurosci 10:77-86. 